ACCEPTED MANUSCRIPT

\title{
Contact/impact modeling and analysis of 4D printed shape memory polymer beams
}

To cite this article before publication: Alireaz Damanpack Moqadam et al 2020 Smart Mater. Struct. in press https://doi.org/10.1088/1361$\underline{665 \mathrm{X} / \mathrm{ab} 883 \mathrm{a}}$

\section{Manuscript version: Accepted Manuscript}

Accepted Manuscript is "the version of the article accepted for publication including all changes made as a result of the peer review process, and which may also include the addition to the article by IOP Publishing of a header, an article ID, a cover sheet and/or an 'Accepted Manuscript' watermark, but excluding any other editing, typesetting or other changes made by IOP Publishing and/or its licensors"

This Accepted Manuscript is @ 2020 IOP Publishing Ltd.

During the embargo period (the 12 month period from the publication of the Version of Record of this article), the Accepted Manuscript is fully protected by copyright and cannot be reused or reposted elsewhere.

As the Version of Record of this article is going to be / has been published on a subscription basis, this Accepted Manuscript is available for reuse under a CC BY-NC-ND 3.0 licence after the 12 month embargo period.

After the embargo period, everyone is permitted to use copy and redistribute this article for non-commercial purposes only, provided that they adhere to all the terms of the licence https://creativecommons.org/licences/by-nc-nd/3.0

Although reasonable endeavours have been taken to obtain all necessary permissions from third parties to include their copyrighted content within this article, their full citation and copyright line may not be present in this Accepted Manuscript version. Before using any content from this article, please refer to the Version of Record on IOPscience once published for full citation and copyright details, as permissions will likely be required. All third party content is fully copyright protected, unless specifically stated otherwise in the figure caption in the Version of Record.

View the article online for updates and enhancements. 

Shape Memory Polymer Beams

\author{
A. R. Damanpack ${ }^{1,2}$, M. Bodaghi ${ }^{2,3}$, W. H. Liao ${ }^{2 \dagger}$ \\ ${ }^{I}$ Department of Technology and Innovation, University of Southern Denmark, Odense, Denmark \\ ${ }^{2}$ Smart Materials and Structures Laboratory, Department of Mechanical and Automation Engineering, \\ The Chinese University of Hong Kong, Shatin, N.T., Hong Kong, China \\ ${ }^{3}$ Department of Engineering, School of Science and Technology, Nottingham Trent University, NG11 8NS \\ Nottingham, United Kingdom \\ $A B S T R A C T$
}

This study examines contact and impact behaviors of shape memory polymer (SMP) beams fabricated by fourdimensional (4D) printing technology. A 3D phenomenological constitutive model is developed to predict viscoelastic-plastic characteristics of SMPs and their shape memory effect in large deformation range for the first time. A novel finite element method (FEM) based on non-linear Green strains is established to analyze the SMP beam under contact/impact loadings. Newmark and Newton-Raphson methods along with an iterative-incremental approach based on a visco-elastic-predictor visco-plastic-corrector return mapping algorithm are implemented to solve FEM governing equations in spatial and time domains. Fused deposition modeling is employed to 4D print samples from polyurethane-based filaments. Thermo-mechanical experimental tests are performed to acquire the parameters needed for the SMP constitutive model. The effects of indentation location, substrate thickness, and edge effect are examined numerically for cylindrical indentation of elastic-plastic SMPs at glassy phase. The validation and application of the Hertzian load-displacement relation for indentation of elastic materials are also clarified. Then, experimental and numerical tests are conducted to examine impact responses of 4D printed SMP beams. Influences of impact position and impactor initial velocity and energy on the responses of the structure in forced and free vibration regimes are studied in detail. The results revealed that the projectile with low velocity or high velocity accompanied with low energy impacted the beam is able to produce plastic deformation. It is shown that the large residual plastic deformation can be fully recovered by simply heating. Due to the absence of similar results in the specialized literature, this paper provides pertinent results that are instrumental in the design of SMP beam-like structures under impact loadings.

\title{
Keywords \\ Contact; Impact; Elastic-plastic; Constitutive modeling; SMP; 4D printing
}

† Correspónding Author. Tel.: +852 3943 8341; fax: +852 26036002.

E-mail address: whliao@cuhk.edu.hk 


\section{Introduction}

The mechanical problem of two deformable bodies in contact/collision has been of great importance in scientific research and technical applications in various fields. Problems related to the contact and impact are complicated and inherently non-linear because of their moving boundaries. Even when friction is considered between the contacting surfaces, the problem becomes more complex as the contact area may exhibit adhesion and unknown slip regions. The first study of the contact problem was made by Hertz (1986) about contact between elastic spherical bodies. It has served as a landmark in the theory of elasticity. The classic Hertz contact theory has been revealed to be successful in replicating the indentation responses for metals and ceramics.

Following the research work by Hertz (1986), contact mechanics on elastic materials has evolved significantly. Many research works have been performed to derive analytical solutions. Mathematical approaches on the basis of complex variables, integral transforms and Green functions have been applied. A comprehensive review of related development can be found in (Hills et al., 1993). A large number of studies have been dedicated to investigate mechanical behaviors of elastic structures under projectile impact based on the contact theories (see e.g., Dunatunga and Kamrin, 2017; Fan et al., 2018; Ivañez et al., 2014; Ranjbar and Feli, 2018). Implementing a finite element method (FEM), Dintwa et al. (2008) examined the validity of the Hertz theory for contact between elastic spheres and contact of an elastic sphere on a rigid substrate. They concluded that the Hertz theory leads significant prediction errors in the large strain regimes. It revealed that the Hertz model underestimates normal force for both cases even at a relatively small indentation. Under large impact, plasticity is expected to be formed first at the impact location and propagates from high-stress level locations along the structure. Elasto-plastic 
dynamic study of structures subjected to impact of projectile involves phenomena such as local contact behavior, wave propagation and structural response (Christoforou and Yigit, 1998). Over the past decades, some theoretical models and dynamic analyses have been devoted to characterize elastic-plastic contact/impact behaviors of homogenous and composite materials and structures (see e.g., Chen et al., 2017; Wang et al., 2017; Zhang et al., 2018).

Shape memory polymers (SMPs) have shown unique capabilities to recover their original shape after undergoing large deformations, on the application of external stimuli, such as temperature. They have been applied in several areas such as aerospace (Liu et al., 2014), and biomedical applications (Fan et al., 2016). SMPs are able to recover large plastic deformation by simply heating. Great progress has also been made in the development of constitutive models and mathematical solutions to describe the SMP behaviors in recent years (see e.g., Akbari et al., 2018; Boatti et al., 2016; Bodaghi et al., 2018, 2019; Ge etal., 2014; Lu et al., 2019; Mao et al., 2015; Pieczyska et al., 2016; Zeng et al., 2018, 2019a,b, 2020). However, to the best of authors' knowledge, their structural responses to contact and impact loadings have been left as a challenge in this field due to the complexity in visco-elastic-plastic behaviors and dynamic simulation. It is also worthwhile to mention that available commercial software is not able to simulate SMPs under static and dynamic loadings.

In 2014, Tibbits (2014) first introduced the concept of four-dimensional (4D) printing structures which are capable of changing their shape and/or function on-demand and over time. 4D printing can be defined as a combination of 3D printing technology and smart materials like SMPs (Bodaghi et al., 2018, 2019; Lin et al., 2019; Liu et al., 2020; Tibbits 2014). Lin et al. (2019) introduced 4D printed biodegradable, remotely controllable and personalized SMP occlusion devices and exemplified atrial septal defect occluders. Liu et al., (2020) investigated anisotropic 
characteristics of mechanical and shape memory performances induced by different infill strategies via both experimental and theoretical methods. SMP samples were 4D printed and uniaxial tensile and compressive tests were performed and simulated by considering generalized MaxwellWiechert model and Prony Series implemented in ABAQUS.

This paper is dedicated to comprehensively investigate contact and impact behaviors of SMPs fabricated by $4 \mathrm{D}$ printing technology. The fourth dimension is related to the shape recovery after plastic deformation (Bodaghi et al., 2018, 2019). First, a node-to-surface algorithm is introduced for global/local searching of the contact. A novel constitutive model is then developed to simulate visco-elastic-plastic behaviors of SMPs and shape memory effect (SME) in the large strain regime. It is then coupled with an FEM formulation. 1D and 2D FEM governing equations are established for the SMP beam under contact/impact loadings for the first time. Newmark method is implemented along with an iterative-incremental Newton-Raphson process based on a viscoelastic-predictor visco-plastic-corrector return mapping algorithm to solve non-linear governing equations in spatial and time domains. Fused deposition modeling (FDM) as a well-known 3D printing technology is implemented to fabricate SMP samples and beams from polyurethane-based filaments. Experiments are conducted to extract thermo-mechanical behaviors of the printed SMPs in the small and large strains. It is shown that the SMP model is capable of replicating experiments well. Mechanical behaviors of the elastic-plastic SMPs at glassy phase indented by a rigid cylindrical indenter are studied experimentally. The effects of indentation location, substrate thickness, and edge effect are investigated. The correctness and reliability of the Hertzian loaddisplacement response for indentation of elastic materials are also checked. Afterwards, impact response of 4D printed SMP beams are studied experimentally and numerically. Influences of impact position and impactor initial velocity and energy on the forced and free vibrational 


\section{Models and Solution Methods}

\subsection{Modeling of contact problem}

Consider two bodies (A and B) as shown in Fig. 1. It indicates the contact between a rigid circular indenter and an SMP body deformed due to the impact. The SMP is assumed to be homogeneous and isotropic with visco-elastic-plastic behaviors as observed in experiments (Guo et al., 2015). Based on the mesh configuration on the contact surface, among conventional contact approaches known as node-to-node, node-to-surface and surface-to-surface contact approaches, the node-to-surface contact search is adopted here. In this approach, the equilibrium is only forced at nodes of one body and compatibility is forced at nodes of the other body. First, a global search is conducted to detect candidate master surfaces from all pre-defined contact surfaces. The contact is then enforced between a slave node and master surface facets local to the node, see Fig. 1. The penetration distance is measured along the normal to the master surface. The parameter $h$ in Fig. 1 shows the depth of penetration of the tip of the indenter into the half space. The method tries to search nodes that violate contact constraint. The contact force is then applied for the violated nodes. A nodal area is assigned to each slave node to convert contact forces to contact stresses. By 
applying contact conditions as well as other boundary conditions, then the nodal displacements are calculated using an iteration approach like Newton-Raphson while the accuracy is satisfied.

\subsubsection{Types of contact interface}

In this paper, the influence of material hardness on the change of surface depth as well as friction coefficient during the elastoplastic deformation have been neglected. Therefore, the relative motion is governed by Coulomb friction model that makes tangent stiffness asymmetric (Zienkiewicz et al., 2014). These contact interface conditions can be expressed mathematically as:

$$
\begin{aligned}
& \text { while } h \geq 0: \quad x_{n}^{\text {slave }}=x_{n}^{\text {master }} \\
& \qquad \begin{array}{l}
\text { if } \frac{\left|F_{t}\right|}{\left|F_{n}\right|}>\bar{\mu} \text { then }\left|F_{t}\right|=\bar{\mu}\left|F_{n}\right| \\
\text { else } x_{t}^{\text {slave }}=x_{t}^{\text {master }}
\end{array}
\end{aligned}
$$

where $x$ and $F$ indicate displacement and force, while the subscripts $t$ and $n$ stand for normal and tangential directions as shown in Fig. 2. Also, $\bar{\mu}$ means the friction, which is an empirical property of the contacting materials. In this study, the slip contact with friction value of 0.4 is assumed.

\subsection{Constitutive SMP model}

\subsubsection{Preliminaries}

Consider an SMP material point in the reference configuration at point $X_{i}$ that moves to a spatial point $x_{i}$. The displacement, $u_{i}$, can be assumed as: 


$$
u_{i}=x_{i}-X_{i}
$$

The deformation gradient, $F_{i j}$, defined as local changes in space of the motion is expressed as:

$$
F_{i j}=\frac{\partial x_{i}}{\partial X_{j}}=\delta_{i j}+\nabla U_{i j}
$$

where $\nabla U_{i j}=\frac{\partial U_{i}}{\partial X_{j}}$. The determinant of the deformation gradient denoted by $J$ (Jacobian of the mapping) can be considered as a measure of the change in volume. The Green deformation tensor is defined as:

$$
C=F^{T} F
$$

It can be expressed in terms of the displacement vector as:

$$
C=I+\nabla U+\nabla U^{T}+\nabla U \nabla U^{T}
$$

The first, second and third invariants of the Green deformation tensor can be given as:

$$
\begin{aligned}
& I_{1}=\operatorname{tr}(C) \\
& I_{2}=\frac{1}{2}\left[(\operatorname{tr}(C))^{2}+\operatorname{tr}\left(C^{2}\right)\right] \\
& I_{3}=J^{2}
\end{aligned}
$$

The Green-Lagrange strain tensor can also be expressed as:

$$
E=\frac{1}{2}(C-I)
$$

where $I$ denotes the second-order identity tensor. 


\subsubsection{Constitutive relations}

SMPs exhibit a combination of hard glassy and soft rubbery phases with elastic-plastic and hyper-elastic behaviors, respectively (Bodaghi et al., 2018, 2019). Volume fractions of these phases are signified by scalar variables of $\zeta_{g}$ and $\zeta_{r}$ satisfying $\zeta_{g}+\zeta_{r}=1$. Subscripts ' $g$ ' and ' $r$ ' indicate glassy and rubbery phases, respectively. The variable $\zeta_{g}$ is considered to be only temperature dependent, i.e., $\zeta_{g}=\zeta_{g}(T)$, as a generally well-known assumption.

By considering the viscosity effect in the dynamic behavior of SMPs (Guo et al., 2015), the glassy phase is assumed to have a visco-elastic-plastic behavior with shape memory feature. On the other hand, the mechanical behavior of the rubbery phase is assumed to be visco-hyper-elastic. Considering that rubbery and glassy phases are linked to each other in a parallel manner, the total deformation gradient, $F$, is multiplicatively decomposed into the visco-elastic, visco-plastic and SME deformation gradients as follows:

$F=F_{g e} F_{g p} F_{g s}=F_{r e}$

where the superscripts $e, p, s$, represent the visco-elastic, visco-plastic, and shape memory components, respectively.

Using Green deformation tensor definition (4), visco-elastic Green deformation tensor of the glassy phase can be derived in terms of total Green deformation tensor as:

$C_{g e}=F_{g p}^{-T} F_{g s}^{-T} C F_{g s}^{-1} F_{g p}^{-1}$

Visco-elastic Green deformation tensor of the rubbery phase can also be expressed as:

$C_{r e}=C$ 
The Green deformation tensor $C$ and the absolute temperature $T$ and visco-plastic Green deformation tensor of the glassy phase $C_{g p}$ are considered as external and internal variables. Assuming the rule of mixtures for the SMP with rubbery and glassy phases, a Helmholtz specific free energy function, $\Theta$, is adopted:

$$
\Theta=\zeta_{g} \Theta_{g}\left(C_{g e}, C_{g p}, T\right)+\left(1-\zeta_{g}\right) \Theta_{r}\left(C_{r e}, T\right)
$$

Free energy functions of rubbery and glassy phases are considered as:

$$
\begin{aligned}
& \Theta_{g}=\frac{1}{\rho_{0}} \Theta_{g e}\left(I_{1 g e}, I_{2 g e}\right)+\frac{1}{\rho_{0}} \Theta_{g t c}\left(I_{1 g}, T\right)+\Theta_{g p}\left(I_{1 g p}, I_{2 g p}\right) \\
& \Theta_{r}=\frac{1}{\rho_{0}} \Theta_{r e}\left(I_{1 r e}, I_{2 r e}, I_{3 r e}\right)+\frac{1}{\rho_{0}} \Theta_{r t c}\left(I_{1 r}, T\right)
\end{aligned}
$$

in which $\rho_{0}$ is the reference density while $I_{1 i j}, I_{2 i j}$ and $I_{3 i j}(i=g, r ; j=e, p)$ signify the first, second and the third invariants of the Green deformation tensor, Adopting Saint-Venant-Kirchhoff viscoelastic strain energy function for the glassy phase (Park et al., 2016), and Mooney-Rivlin viscohyper-elastic strain energy function for the rubbery phase (Alwan and Hamza, 2010), $\Theta_{g e}$ and $\Theta_{r e}$ are written as:

$$
\begin{aligned}
& \Theta_{g e}=c_{1 g}\left(I_{1 g e}-3\right)^{2}+c_{2 g}\left(2\left(I_{1 g e}-3\right)-\left(I_{2 g e}-3\right)\right)+\mu_{g} \dot{C}: C \\
& \Theta_{r e}=c_{1 r}\left(I_{3 r e}^{-1 / 3} I_{1 r e}-3\right)+c_{2 r}\left(I_{3 r e}^{-2 / 3} I_{2 r e}-3\right)+c_{3 r}\left(I_{3 r e}^{1 / 2}-1\right)^{2}+\mu_{r} \dot{C}: C
\end{aligned}
$$

where $c_{i}(i=1,2,3)$ are material constants while $\mu$ is viscosity parameter. Visco-plastic configurational energy of the glassy phase is also expressed as:

$$
\left.\Theta_{g p}=\frac{1}{8} h_{g p}\left(\left(I_{1 g p}\right)-3\right)^{2}+4\left(I_{1 g p}-3\right)-2\left(I_{2 g p}-3\right)\right)+\mu_{g p} \dot{C}_{g p}: C_{g p}
$$

where $h_{g p}$ and $\mu_{g p}$ are hardening and viscosity parameters of the plastic deformation in the glassy

phase. Furthermore, thermo-chemical energies of $\Theta_{i t c}(i=g, r)$ are: 


$$
\Theta_{i t c}=-\frac{3}{2} \alpha_{i} \kappa_{i}\left(I_{1 i}-3\right)\left(T-T_{0}\right)+u_{0 i}-T s_{0 i}+c_{i}\left[\left(T-T_{0}\right)-T \ln \left(T / T_{0}\right)\right]
$$

where $\alpha_{i}$ is the thermal expansion coefficient; $\kappa_{i}$ is the bulk modulus; $u_{0 i}$ and $s_{0 i}$ signify the specific internal energy and entropy at the equilibrium temperature $T_{0}$; and $c_{i}$ denotes the specific heat at constant volume. Then, the Clausius-Duhem inequality is written to derive constitutive relationship as:

$$
D_{m}=\frac{1}{2 \rho_{0}} S: \dot{C}-(\dot{\Theta}+s \dot{T}) \geq 0
$$

where $S$ and $S$ are the second Piola-Kirchhoff stress tensor and the specific entropy. By substituting the time derivative of the Helmholtz free energy (11) into the mechanical dissipation inequality (16), state equations can be derived as:

$$
\begin{aligned}
& S=\zeta_{g} S_{g}+\left(1-\zeta_{g}\right) S_{r} \\
& S=\zeta_{g} S_{g}+\left(1-\zeta_{g}\right) S_{r}
\end{aligned}
$$

The Clausius-Duhem inequality (16) is also simplified to

$$
X_{g p}: \dot{C}_{g p} \geq 0
$$

where $X_{g p}$ represents the dissipative force related to the plastic velocity gradient.

\subsubsection{Evolution laws and solutions}

The model is completed by defining evolution equations for $C_{g p}, \zeta_{g}$ and $F_{g s}$. To satisfy the Clausius-Duhem inequality during plastic deformation of the glassy phase, the evolution law for $C_{g p}$ is adopted as: 


$$
\dot{C}_{g p}=\dot{\omega} X_{g p}^{D} /\left\|X_{g p}^{D}\right\|
$$

where $\omega$ denotes non-negative consistency parameter and superscript of $D$ makes the component deviatoric. Introducing Eq. (19) into the Clausius-Duhem inequality (18) results in:

$$
\dot{\omega}\left\|X_{g p}^{D}\right\| \geq 0
$$

The following limit function is given to evolve the plastic deformation into the glassy phase.

$$
L_{g p}=\left\|X_{g p}^{D}\right\|-Y_{g p}
$$

where the constant parameter of $Y_{g p}$ governs the kinetics of plastic evolution. The Kuhn-Tucker conditions are also met to constrain the evolution of $C_{g p}$ as:

$$
L_{g p} \leq 0, \dot{\omega} \geq 0, L_{g p} \dot{\omega}=0
$$

Thermal conditions for the plastic deformation evolution of the glassy phase and its recovery are assumed as:

$$
\left\{\begin{array}{l}
\text { if }\left(T \leq T_{l}\right) \text { or }\left(T_{l}<T<T_{h}, \dot{T} \leq 0\right) \text { then } C_{g p} \text { evolves via Eq. (22) } \\
\text { if }\left(T_{l}<T<T_{h}, \dot{T} \geq 0\right) \text { then } C_{g p} \text { remains constant } \\
\text { if }\left(T \geq T_{h}\right) C_{g p} \text { is set } I
\end{array}\right.
$$

It states that plastic deformation evolves mechanically at low temperatures $\left(T \leq T_{l}\right)$ or during cooling in the transition regime $\left(T_{l}<T<T_{h}\right)$ while it is recovered by heating within transition range.

Evolution of SME deformation gradient in the glassy phase is given as:

$$
\left\{\begin{array}{l}
\text { if }\left(T \geq T_{h}\right) \text { then } F_{g s}=c_{g s}(F-I)+I \\
\text { otherwise } F_{g s} \text { remains constant }
\end{array}\right.
$$


where $c_{g s}$ ranging within [ $\left[\begin{array}{ll}0 & 1\end{array}\right]$ controls the shape-fixing imperfection in shape memory programming. Finally, the evolution of $\zeta_{g}$ during glassy-rubbery phase transformation can be formulated as:

$$
\zeta_{g}=-\frac{\tanh \left(\gamma_{1} T_{g}-\gamma_{2} T\right)-\tanh \left(\gamma_{1} T_{g}-\gamma_{2} T_{h}\right)}{\tanh \left(\gamma_{1} T_{g}-\gamma_{2} T_{h}\right)-\tanh \left(\gamma_{1} T_{g}-\gamma_{2} T_{l}\right)}
$$

where parameters $\gamma_{1}$ and $\gamma_{2}$ are chosen to match the experimental DMA curve.

Finally, in conjugation with the solution of the developed constitutive model, it can be solved by performing an incremental-iterative scheme based on the elastic-predictor plastic-corrector return mapping method details of which can be found in Simo and Hughes (1998). A visco-elastic trial state is considered for $C_{g p}$ and a trial value of the limit function (21) is calculated to check for the trial state admissibility. If the latter is not verified, the step is visco-plastic and the evolution equation (19) is integrated. It is discretized via the explicit Forward-Euler integration and solving non-linear system of algebraic equations by means of iterative Newton-Raphson method (Reddy, 2004; Simo and Hughes, 1998).

\subsection{Finite element formulation}

In order to extract the governing equations of motion for the SMP body under impact loading, the Hamilton principle is used as:

$$
\int_{t 1}^{t 2}\left(\delta W_{e}-\int_{\forall}\left(\delta W_{i}-\delta K\right) d \forall\right) d t=0
$$


where $\forall$ signifies the body volume and $W_{e}$ is the work done by external loads. Furthermore, $W_{i}$ and $K$ are thermo-visco-elastic and kinetic energies defined as:

$$
\begin{aligned}
& \int_{t 1}^{t 2} \int_{\forall} \delta W_{i} d \forall d t=\int_{t 1}^{t 2} \int_{\forall} \delta C: \frac{\partial W_{i}}{\partial C} d \forall d t=\int_{t 1}^{t 2} \int_{\forall} \frac{1}{2} \delta C: S d \forall d t \\
& \int_{t l}^{t 2} \int_{\forall} \delta K d \forall d t=\int_{t l}^{t 2} \int_{\forall} \delta \dot{U} \cdot \dot{U} \rho d \forall d t=-\int_{t l}^{t 2} \int_{\forall} \delta U \cdot \ddot{U} \rho d \forall d t
\end{aligned}
$$

Considering zero initial conditions, Eq. (26) can be written as:

$$
\int_{\forall}\left(\frac{1}{2} \delta C: S\right) d \forall+\int_{\forall}(\delta U . \ddot{U}) \rho d \forall=\delta W_{e}
$$

Assume a general beam element with length $l$, width $b$ and thickness $h$, as shown in Fig. 3. A local 2D Cartesian coordinate system $(X, Z)$ is located on the beam element. An appropriate kinematic hypothesis for the present beam under contact/impact loading is the 1D and 2D plane stress assumption. It implies:

$$
\begin{aligned}
& 1 D:\left\{\begin{array}{l}
S_{22}=S_{33}=S_{23}=S_{12}=0 \\
\lambda_{1}=C_{22}, \lambda_{2}=C_{33}, \lambda_{3}=C_{23}, \lambda_{4}=C_{12}
\end{array}\right. \\
& 2 D:\left\{\begin{array}{l}
S_{22}=S_{23}=S_{12}=0 \\
\lambda_{1}=C_{22}, \lambda_{2}=C_{23}, \lambda_{3}=C_{12}
\end{array}\right.
\end{aligned}
$$

where $\lambda_{i}(i=1 . .4)$ are the Lagrange multiplier coefficient.

In order to solve the problem, an FE method is implemented. For the 1D case, a finite-strain beam model is considered to describe the displacement field in the beam domain. It is formulated as: 


$$
\begin{aligned}
& V(X)=v(X)-Z \sin (\beta(X)) \\
& W(X)=-Z+Z \cos (\beta(X))+w(X)
\end{aligned}
$$

where $V$ and $W$ indicates displacement components of a material point within the element along $X$ and $Z$ directions. Also, $v$ and $w$ denote mid-plane displacements along $X$ and $Z$ coordinates while $\beta$ is cross-section rotation.

Quadratic Lagrange shape functions are employed to interpolate mechanical variables, $\phi$, in terms of nodal variables, $\phi_{i}(i=1,2,3)$, as shown in Fig. 3 as:

$$
\phi=N(\eta) \bar{\phi}
$$

where

$$
\begin{aligned}
& N=\left[\begin{array}{lll}
\frac{1}{2} \eta(\eta-1) & 1-\eta^{2} & \frac{1}{2} \eta(\eta+1)
\end{array}\right], \quad \eta=2 X / l \\
& \bar{\phi}=\left[\begin{array}{lll}
\phi_{1} & \phi_{2} & \phi_{3}
\end{array}\right]^{T}
\end{aligned}
$$

For the case of $2 \mathrm{D}$ beam model, a three-nodded triangular element in the $X-Z$ plane is used to discretize the domain. Lagrangian multiplier is interpolated using linear shape functions with continuity of $C^{0}$ as shown in Fig. 4. The linear interpolation can be expressed as:

$$
\phi=L(\eta, \xi) \bar{\phi}
$$

in which $L$ and $\bar{\phi}$ are linear shape function and nodal variable vector defined as:

$$
\begin{aligned}
& L=\left\{\begin{array}{lll}
1-\eta-\xi & \eta
\end{array}\right\} \\
& \bar{\phi}=\left\{\begin{array}{lll}
\phi_{1} & \phi_{2} & \phi_{3}
\end{array}\right\}^{T}
\end{aligned}
$$

where $\eta, \xi$ are natural local coordinates while $\phi_{1}, \phi_{2}, \phi_{3}$ are nodal variables. Adopting a linear natural coordinate system for the triangular element, the derivative of Cartesian coordinates can also be obtained as: 


$$
\left\{\begin{array}{l}
d X \\
d Z
\end{array}\right\}=[\bar{J}]\left\{\begin{array}{l}
d \eta \\
d \xi
\end{array}\right\}
$$

where

$$
\bar{J}=\left[\begin{array}{ll}
X_{2}-X_{1} & Z_{2}-Z_{1} \\
X_{3}-X_{1} & Z_{3}-Z_{1}
\end{array}\right]
$$

The elemental displacements are also interpolated by means of Hermitian shape functions with continuity of $C^{1}$ as illustrated in Fig. 5. For instance, typical displacement like $\Phi$ can be expressed as:

$$
\Phi=N(\eta, \xi) \bar{\Phi}
$$

in which

$$
\begin{aligned}
N & =\left[\begin{array}{lllllllll}
N_{1} & M_{1} & O_{1} & N_{2} & M_{2} & O_{2} & N_{3} & M_{3} & O_{3}
\end{array}\right] \\
\Phi & =\left[\begin{array}{lllllllll}
\Phi_{1} & \Phi_{1, X} & \Phi_{1, Z} & \Phi_{2} & \Phi_{2, X} & \Phi_{2, Z} & \Phi_{3} & \Phi_{3, X} & \Phi_{3, Z}
\end{array}\right]^{T}
\end{aligned}
$$

where $N_{i}, M_{i}, Q_{i}(i=1,2,3)$ are Hermitian shape functions details of which can be found in (Reddy, 2004). The derivative of global displacements can be expressed in terms of their local counterparts as:

$$
\left\{\begin{array}{l}
\Phi_{i, X} \\
\Phi_{i, Z}
\end{array}\right\}=[\bar{J}]^{-1}\left\{\begin{array}{l}
\Phi_{i, \eta} \\
\Phi_{i, \xi}
\end{array}\right\} \quad i=1,2,3
$$

Therefore, the elemental local displacements and Lagrangian multiplier can be interpolated in terms of nodal variables through shape functions as:

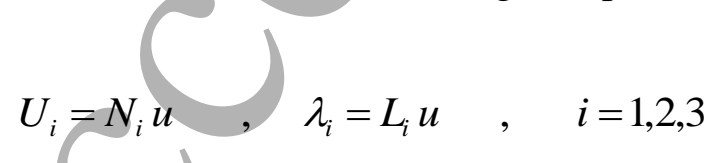

The derivative of the elemental displacements can be derived as: 


$$
\nabla U_{i j}=\frac{\partial N_{i}}{\partial X_{j}} u \quad(i, j=1,2,3)
$$

Derivative of $C$ and $S$ can also be expressed in the discretized form as:

$$
\begin{aligned}
& \frac{\partial C_{i j}}{\partial u}=\bar{N}_{i j}(u) \quad(i, j=1,2,3) \\
& \frac{\partial S_{i j}}{\partial u}=\bar{M}_{i j}(u) \quad(i, j=1,2,3)
\end{aligned}
$$

Finally, by substituting Eqs. (40) and (42) into the Hamilton principle (28), the finite element governing equations for a base element can be derived as:

$$
\int_{\forall}\left(\frac{1}{2} \bar{N}_{i j}^{T} S_{i j}\right) d \forall+\left(\int_{\forall} N_{i}^{T} N_{i} \rho d \forall\right) \ddot{u}=f
$$

where $f$ is the mechanical force vector. Eq. (43) is a highly non-linear equation in terms of mechanical nodal variables and elemental stress in the time domain. It can be expressed as:

$$
f_{k}(u)+\in(u) \dot{u}+M \ddot{u}-f=0
$$

where $\epsilon$ and $A$ are damping and mass matrices. In order to find a solution of the present contact problem with geometric and material non-linearities, an iterative approach such as NewtonRaphson (Reddy, 2004) is implemented. To this end, a residual vector is first introduced as:

$$
R=f_{k}(u)+C(u) \dot{u}+M \ddot{u}-f
$$

By using the implicit time integration scheme of Newmark method, the time derivatives appeared in (45) are approximated as:

$$
\begin{aligned}
& \dot{u}_{t+\Delta t}=\frac{1}{2 \theta \Delta t} u_{t+\Delta t}-\left(\frac{1}{2 \theta \Delta t} u_{t}+\frac{1-2 \theta}{2 \theta} \dot{u}_{t}+\frac{(1-4 \theta) \Delta t}{4 \theta} \ddot{u}_{t}\right)=\frac{1}{2 \theta \Delta t} u_{t+\Delta t}-\dot{\bar{u}}_{t} \quad 0 \leq \theta \leq 0.5 \\
& \ddot{u}_{t+\Delta t}=\frac{1}{\theta \Delta t^{2}} u_{t+\Delta t}-\left(\frac{1}{\theta \Delta t^{2}} u_{t}+\frac{1}{\theta \Delta t} \dot{u}_{t}+\frac{1-2 \theta}{2 \theta} \ddot{u}_{t}\right)=\frac{1}{\theta \Delta t^{2}} u_{t+\Delta t}-\ddot{\bar{u}}_{t}
\end{aligned}
$$


resulting in:

$$
R_{t+\Delta t}=f_{k}\left(u_{t+\Delta t}\right)+C\left(u_{t+\Delta t}\right) \dot{u}_{t+\Delta t}+M \ddot{u}_{t+\Delta t}-f_{t+\Delta t}
$$

Then, equilibrium is reached for each time step by implementing Newton-Raphson iteration until a convergence criterion is met. The residual vector (47) is rewritten for the $n^{\text {th }}$ iteration as:

$$
R_{\left\{\begin{array}{l}
t+\Delta t \\
n
\end{array}\right.}=f_{k}\left(u_{\left\{\begin{array}{l}
t+\Delta t \\
n
\end{array}\right.}\right)+E\left(u_{\left\{\begin{array}{l}
t+\Delta t \\
n
\end{array}\right.}\right)\left(\frac{1}{2 \theta \Delta t} u_{\left\{\begin{array}{l}
t+\Delta t \\
n
\end{array}\right.}-\dot{\bar{u}}_{t}\right)+M\left(\frac{1}{\theta \Delta t^{2}} u_{\left\{\begin{array}{l}
t+\Delta t \\
n
\end{array}\right.}-\ddot{\bar{u}}_{t}\right)-f_{t+\Delta t}
$$

The tangent matrix defined as differentiation of $R$ with respect to the nodal displacement vector $\boldsymbol{u}$ is derived as:

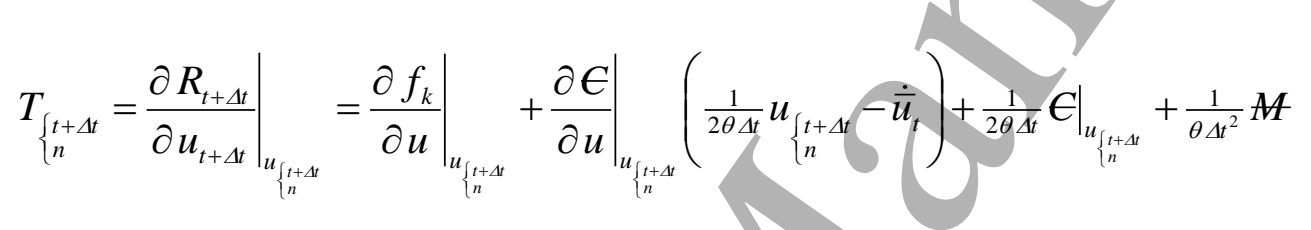

The updated value of the nodal displacement vector can be calculated as:

$$
u_{\left\{\begin{array}{l}
t+\Delta t \\
n+1
\end{array}\right.}=u_{\left\{\begin{array}{l}
t+\Delta t \\
n
\end{array}\right.}-T_{\{\begin{array}{l}
t+\Delta t \\
n
\end{array} \underbrace{-1}_{\{t} R^{n+\Delta t}}
$$

Finally, Eqs. (44) and (49) are used to produce global FE equations and the global tangent matrix by assembling and applying boundary conditions. The overall non-linear algebraic equations are solved by means of the iterative Newton-Raphson method (Reddy, 2004).

As the dynamic force induced by impact loading is unknown, it is needed to define it to complete the dynamic analysis. Fig. 6 shows a schematic of the pendulum impact test system. It consists of a pendulum arm with length $R_{e}$ and weight $M$, bolted at distance of $r$ and an impactor fixed at the end of the arm. The impact pendulum can generate impact loading throughout the collision at adaptable velocity and consequently kinetic energy by adjusting the weight position and starting height, $h$ ( $h=R_{e}$ when the pendulum arm hangs straight down at rest). Considering 
$M=0.4686 \mathrm{~kg}$ plus mass of the arm and impactor and the range of $0.15-0.45 m$ for $r$, the adjustable pendulum impactor can be equivalent with a single mass located at radius of $R_{m}=0.0865+0.624 \mathrm{rm}$ with respect to the hinge. The equivalent moment of inertia is calculated as $J_{0}=0.0148+0.292 r^{2} \mathrm{~kg} \cdot \mathrm{m}^{2}$. The maximum velocity of the impactor just before collision can be computed via:

$$
V_{o}=\sqrt{2 M \cdot g \cdot R_{m} \cdot R_{e}\left(R_{e}-h\right) / J_{o}}
$$

The impactor is lifted to a desired height and then released to impact on the beam, see Figs. 6 and 7. Indicating maximum angles of the impactor and the beam after the collision, respectively, by $\psi_{1}$ and $\psi_{2}$, maximum kinetic energy of the pendulum mass can be defined as:

$$
J_{0} \ddot{\psi}_{1}=-P R_{e} \cos \left(\psi_{1}-\psi_{2}\right)
$$

This equation is coupled with Eq. (44) and completes the FEM governing equations of the present problem. In order to solve the dynamic problem, the following initial conditions are considered:

$$
\begin{array}{ll}
\{u\}_{t=0}=\{0\} & \{\dot{u}\}_{t=0}=\{0\} \\
\dot{\psi}_{1 t=0}=0 & \dot{\psi}_{1 t=0}=V_{0} / R_{e}
\end{array}
$$

\section{Materials and Fabrication}

Material properties presented in the model are determined in this section. Samples are designed using the 3D CAD program Solidworks and printed using a FlashForge New Creator Pro 3D printer. The nozzle diameter is $0.4 \mathrm{~mm}$. Commercial SMP materials (filament with $1.75 \mathrm{~mm}$ in diameter) are selected to fabricate samples. Printing parameters such as layer height and temperatures of nozzle extrusion, build tray and chamber are selected to be as $0.2 \mathrm{~mm}$ and 230, 50 
and $25^{\circ} \mathrm{C}$ while printing speed is $10 \mathrm{~mm} / \mathrm{s}$. Samples are printed with $100 \%$ infill with parallel line and one shell layer.

The parameters of the SMP phase transformation like $\gamma_{1}, \gamma_{2}$ and $T_{l}, T_{h}, T_{g}$ in Eq. (25) are measured via a DMA test (Model 242, NETZSCH) in axial tension mode. DMA samples are printed with dimensions of $16 \times 1.6 \times 1 \mathrm{~mm}$. The test is conducted with ratio of dynamic stress to static stress equals to 1.5 , frequency of force oscillation $1 \mathrm{~Hz}$, and heating ramp of $5^{\circ} \mathrm{Cmin}{ }^{-1}$ within $[-20 . .85]^{\circ} \mathrm{C}$. Fig. 8 shows experimental thermo-mechanical behavior of the printed SMP in terms of storage modulus, $E_{s}$, and phase lag, $\tan (\delta)$. The parameters of $\gamma_{1}, \gamma_{2}$ and $T_{l}, T_{h}, T_{g}$ are calibrated using DMA data as listed in Table 1. Experimental elastic modulus is compared with numerical one calculated based on the defined parameters in Fig. 9. It is seen that Eq. (25) can smoothly produce phase transformation that is in a good agreement with experiments.

Next, elastic material properties of $c_{1 g}, c_{2 g}, \alpha_{g}, \kappa_{g}, c_{g f}$ introduced in 2.2.2 and 2.2.3 sections are determined via small-strain tensile and thermal tests. In this respect, a Tinius Olsen ${ }^{\circledR} \mathrm{H} 5 \mathrm{kS}$ (Tinius Olsen, Horsham, PA, USA) uniaxial testing machine with a $5 \mathrm{kN}$ load cell is utilized. Material parameters of $\rho_{0}, h_{g p}, c_{1 r}, c_{2 r}, Y_{g p}$ introduced in 2.2.2 and 2.2.3 sections are also calibrated as listed in Table 1 by uniaxial large-strain experimental tensile tests conducted at 23 and $85^{\circ} \mathrm{C}$ as shown in Fig. 10. Samples have been printed according to the geometry and dimensions described by ASTM D638-10 (2010). Modeling results based on the defined parameters are included in Fig. 10 for a full loading-unloading cycle. The results presented in Fig. 10 reveal that the constitutive model is able to well replicate elastic-plastic and hyper-elastic behaviors of SMPs at low and high temperatures. 


\section{Structural Analysis}

In this section, mechanical behaviors of the printed SMPs under contact and impact loadings are investigated numerically and experimentally. 2D finite element model is implemented for analyzing contact of the printed SMP substrates. Then, 1D and 2D finite element models are applied to investigate impact responses of the SMP beams.

\subsection{Contact study}

The accuracy and reliability of the Hertzian load-displacement response for indentation of materials are checked here. As the first example, the rigid cylindrical indentation $(R=1 \mathrm{~mm})$ on an elastic SMP substrate with dimension of $(L=b=100 \mathrm{~mm})$ and various thickness $(H=1 \ldots 100 \mathrm{~mm})$ is studied numerically as shown in Fig. 11 . The substrate can be considered as a beam with stiffness of SMP glassy phase $\left(E=1.6 \mathrm{GPa}, v=0.45, \mathrm{~T}=23^{\circ} \mathrm{C}\right)$ with fixed bottom face contacted at different positions $(X i)$. Fig. 12 depicts the results in terms of contact stiffness $(K)$ defined as force over indentation for different contact positions $(X i / R)$. It is seen that the contact stiffness reduces as the substrate becomes thicker. It can be found that, for thin substrates, the contact stiffness mostly remains unchanged in the middle of the substrate, while it drops down drastically in the edge area. However, this variation becomes smooth and gradually happens as the substrate thickness is increased. This figure challenges the validity of the Hertz theory, which is widely employed to predict load-indentation response of elastic thin/thick structures, see e.g. (Ranjbar and Feli, 2018). As it can be concluded, the Hertz theory is not valid to be used in the edge area. The results are also presented in different way in Fig. 13 in terms of contact stiffness versus substrate thickness at the edge and middle positions $(X i / R=0,50)$. It is seen that the 
Next, static mechanical behavior of an elastic-plastic SMP substrate with material properties detailed in Table 1 and geometrical parameters of $(L=20 \mathrm{~mm}, b=10 \mathrm{~mm}, H=1 \mathrm{~mm})$ in contact with a cylindrical rigid indenter with radius of $R=1 \mathrm{~mm}$ is investigated numerically. It is assumed that the SMP substrate is at glassy phase in the low temperature of $T=23^{\circ} \mathrm{C}$. The load per unit width against indentation $(F / b-w)$ at the edge and center of the SMP substrate is demonstrated in Fig. 15. As it can be seen, the indentation load-displacement at tip point is lower than its counterpart at the middle zone that is predictable. It is also observed that the relationship between indentation load and displacement is linear in the elastic range $(\Delta w=0 \ldots 8 \mu m)$ beyond which it becomes non-linear. This non-linear behavior happens when the SMP material experiences plasticity. As it can be seen, there are two non-linear segments in the plastic domain. While there is a softening response within the range of $\Delta w=8 \ldots 12 \mu m$, the material reveals an instant hardening at $\Delta w=12 \mu m$ that changes the slop of the load-displacement drastically. This can be due to strain hardening effect and/or the increase in the contact area with elastic behaviors. Fig. 15 also shows that the difference between indentation load-displacement curves increases as indentation depth becomes deeper. Finally, variation of contact stiffness ratio defined as $K(X i=0) / K\left(X i=\frac{1}{2} L\right)$ along the length of the SMA beam is illustrated in Fig. 16. The results in Figs. 15 and 16 obtained from 2D FEM contact modeling can serve as benchmark for impact analysis of the SMP beam using 1D impact model investigated in the following section. By knowing the contact position, the 
contact stiffness can be extracted and applied to the impact analysis implementing 1D FEM model rather than a $2 \mathrm{D}$ one. This approach can reduce the computational cost and complexity.

\subsection{Impact analysis}

This section is dedicated to dynamic response of a 4D printed SMP beam with material properties detailed in Table 1 and dimension of $L=20 \mathrm{~mm}, b=10 \mathrm{~mm}, H=1 \mathrm{~mm}$ subjected to an impact by a $1 \mathrm{~mm}$ diameter rigid impactor striking with the initial velocity of $V_{0}=1,2,2.5 \mathrm{~m} / \mathrm{s}$ at $X i=0, \frac{1}{5} \mathrm{Lmm}$. In all case studies, unless otherwise stated, the value of moment of inertia is set as $J i=0.0243 \mathrm{kgm}^{2} .1 \mathrm{D}$ and 2D FEM impact models are implemented to analyze the problem. Regarding 1D FEM model, the contact stiffness presented in Figs. 15 and 16 are adopted. Experiments are also conducted to verify the accuracy of the developed models. A Photron FASTCAM Mini UX 50 high-speed video camera is mounted on a tripod facing the printed beam. Displacement of the beam and velocity of the impactor are measured using the recordings during forced and free vibration regimes.

Fig. 17 ad shows computational time history of non-dimensional displacement $(W / H)$, impactor velocity, contact force, and energy of the SMP beam impacted by $V_{0}=1 \mathrm{~m} / \mathrm{s}$ at its tip point, $X i=0$. Experimental results related to the displacement and velocity are also included in Fig. 17a and 17b. Moreover, configuration of the beam captured experimentally in the forced vibration range is compared with that of 2D FEM in Fig. 18. The results presented in Figs. 17 and 18 show that $1 \mathrm{D}$ and $2 \mathrm{D}$ model results for the maximum and residual displacement of the beam, impactor velocity and forced-vibration configuration of the beam agree well with those from the experimental testing. For example, both $1 \mathrm{D}$ and $2 \mathrm{D}$ models predict the maximum experimental displacement with $4.8 \%$ error. It is seen that the maximum beam displacement and contact force 
occur at about $9 m s$ when the impactor velocity becomes zero and the beam starts changing its motion direction. At the peak point, the kinetic energy of the impactor fully transfers to the beam and the strain energy becomes maximum while some energy is dissipated. It should be pointed out that the sum of the kinetic energy of the impactor and strain and kinetic energies of the beam plus dissipated energy is always constant and equal to the initial kinetic energy of the impactor. As it can be found from Fig. 17d, the structure gets a low kinetic energy in this low-speed case study. Once the motion direction changes, strain energy and displacement of the beam plus contact force decrease while the kinetic energy and velocity of the impactor and dissipated energy of the beam are increased. It is seen that the impactor with maximum kinetic energy and velocity leaves the beam at about $18 \mathrm{~ms}$. The structure then starts vibrating freely around $W / H=0.2$ with a low level of the strain energy. It is observed that the free vibration phase decays at approximately $30 \mathrm{~ms}$, while a small plastic strain remains into the beam.

The effect of impactor initial velocity is investigated in the next example. The previous case was considered for initial velocity of $V_{0}=2 \mathrm{~m} / \mathrm{s}$. The counterpart of Figs. 17 and 18 for the present example is demonstrated in Figs. 19 and 20. The preliminary conclusion is that 2D FEM model replicates maximum displacement and impactor velocity more accurately compared to the $1 \mathrm{D}$ one. For example, while 2D model predicts the maximum experimental displacement with $4.3 \%$ error, the $1 \mathrm{D}$ one underestimates the maximum displacement as large as $12.5 \%$. Comparing the results for different initial velocities presented in Figs. 17-20, the faster impactor is able to increase the maximum displacement up to $40 \%$ and reduce the impact time up to $50 \%$. It even produces a contact force that is $280 \%$ larger than one produced by $V_{0}=1 \mathrm{~m} / \mathrm{s}$. As it can be seen in Fig. 20, this contact force is so large enough that the impactor passes the beam at $4.5 \mathrm{~ms}$. During forced vibration regime, the impactor partially transfers its kinetic energy to the beam, see Fig. 19d. It is 
observed that the strain energy of the beam becomes maximum while a small amount of the energy is dissipated and low absorbed kinetic energy almost remains unchanged. Once the impactor passes the beam and leaves it, the structure starts oscillating freely and elastically via conversion between strain energy and kinetic energy, see Fig. 19a and 19d. As it can be seen, while the beam dissipates the energy, the vibration amplitude attenuates so considerably that free vibration phase decays at approximately $30 \mathrm{~ms}$. Finally, by focusing on value of the vibration amplitude when the impactor leaves the beam, shown in Figs. 17a and 19a, it can be noticed that the oscillation amplitude in the free motion phase depends on the distance from the equilibrium condition at the end of forced motion regime.

Influence of impactor initial velocity is further studied by conducting numerical and experimental tests with initial velocity of $V_{0}=2.5 \mathrm{~m} / \mathrm{s}$. The counterpart of Figs. 19a and $19 \mathrm{~b}$ is demonstrated in Figs. 21a and $21 \mathrm{~b}$ for the present case study. The results presented in Figs. 19 and 21 reveal that, increasing the impactor velocity to $2.5 \mathrm{~m} / \mathrm{s}$ that is $25 \%$ higher than the previous test reduces the impact time up to $22 \%$ while not affecting the maximum amplitude significantly. It is due to the fact that the structure does not have time enough to be deformed during the impact. It is also seen that the high-yelocity impact does not produce any plastic deformation so that the structure vibrates freely and elastically around the initial equilibrium state $W / H=0$.

Next, the effect of the impactor position is examined. The SMP beam is impacted by a projectile with velocities of $V_{0}=1 \mathrm{~m} / \mathrm{s}$ at position of $X i=\frac{1}{5} \mathrm{~L}$. The experimental and numerical results in terms of time history of displacement and impactor velocity are depicted in Fig. 22. It is seen that the 2D FEM can replicate the experiment very well. Comparing results with their counterpart in Fig. 17 shows that, impacting at $X_{i}=\frac{1}{5} L$ does not affect maximum displacement in free and forced 
vibration, plastic deformation and maximum velocity of the impactor when leaving the beam. However, due to the higher local bending rigidity in the vicinity of the clamped edge of the beam, impacting at $X_{i}=\frac{1}{5} L$ reduces the impact time up to $33 \%$.

The effect of the impactor position is further investigated for the high-velocity impact experimentally and numerically. Fig. 23a and c demonstrates time history of displacement and impactor velocity for the case of $V_{0}=2.5 \mathrm{~m} / \mathrm{s}$ and $X i=\frac{1}{5} \mathrm{~L}$. Shape recovery under temperature control is also studied in Fig. 23b via heating the deformed static beam at $T=20{ }^{\circ} \mathrm{C}$ to $100{ }^{\circ} \mathrm{C}$ and then cooling down to $T=20^{\circ} \mathrm{C}$. Moreover, configuration of the beam captured experimentally and numerically in the first $30 \mathrm{~ms}$ is illustrated in Fig. 24. The primary conclusion drawn from Figs. 23 and 24 is that the 2D FEM model can replicate experiment well. The results in Fig. 21 show that the projectile with high velocity of $V_{0}=2.5 \mathrm{~m} / \mathrm{s}$ impacted the tip point passes the beam. However, Figs. 23 and 24 reveal that, when the projectile with the same velocity is impacted at $X_{i}=\frac{1}{5} L$, it slides on the top surface of the structure at the first $17 \mathrm{~ms}$. It moves toward the tip of the beam and then gets back to the initial impact position, and finally leaves the beam. As the impact time lasts $370 \%$ longer than impact on the tip point, the structure experiences maximum displacement of $W / H=6.2$ that is $82 \%$ larger than the maximum displacement induced by the impact on the tip point. It is even seen that a tip displacement as large as $W / H=1.2$ remains into the beam due to the higher local bending rigidity at $X_{i}=\frac{1}{5} L$. It is worth mentioning that, although displacement value and impact time are different for two cases, the velocity of the leaving impactor is similar for both cases. It can also be seen from Fig. $23 \mathrm{~b}$ that the large residual plastic deformation can be fully recovered by simply heating. Finally, when the results presented in Fig. 23 are compared with those in Fig. 22, it can be found that, increasing $V_{0}$ 
from $1 \mathrm{~m} / \mathrm{s}$ to $2.5 \mathrm{~m} / \mathrm{s}$ enhances the maximum displacement up to $139 \%$. The impact time lasts $30 \%$ longer as well.

Finally, the effects of moment of inertia on the impact response of the SMP beam are investigated. As calculated in section 2.3, the moment of inertia directly depends on the distance of the mass from the reference point. The SMP beam is impacted at position of $X i=0, \frac{1}{5} L$ by a projectile with velocity of $V_{0}=1,2.5 \mathrm{~m} / \mathrm{s}$ but different values of moment of inertia $J_{0}=0.0243,0.0440,0.0645 \mathrm{kgm}^{2}$. The counterpart of Figs. 19a and 19c is demonstrated in Figs. 25-28 for the cases of $V_{0}=1 \mathrm{~m} / \mathrm{s}, X i=0 ; \quad V_{0}=1 \mathrm{~m} / \mathrm{s}, X i=\frac{1}{5} L ; \quad V_{0}=2.5 \mathrm{~m} / \mathrm{s}, X i=0 ; \quad$ and $V_{0}=2.5 \mathrm{~m} / \mathrm{s}, X i=\frac{1}{5} L$, respectively. The preliminary conclusion drawn from these figures is that the projectile with low velocity or high velocity accompanied with low energy impacted the beam at $X i=\frac{1}{5} L$ is able to produce plastic deformation while other three cases make the beam vibrate elastically. From Fig. 25, it is concluded that, while the projectile with low velocity and moment of inertia $\left(V_{0}=1 \mathrm{~m} / \mathrm{s}, J_{0}=0.0243 \mathrm{Kgm}^{2}\right)$ impacted the tip point of the beam cannot pass the structures, in other two cases with higher moment of inertia, the impactor is able to pass the beam. It is also seen that the projectile with characteristics of $V_{0}=1 \mathrm{~m} / \mathrm{s}, J_{0}=0.0243 \mathrm{Kgm}^{2}$ makes a halfsine-like impact pulse and forced displacement, while any increase in the moment of inertia changes them to exponential ones with larger magnitude. It is worth mentioning that, while the increase from $J_{0}=0.0440 \mathrm{Kgm}^{2}$ to $0.0645 \mathrm{Kgm}^{2}$ makes the impact time shorter, it does not affect the maximum impact force and displacement.

The results in Fig. 26 reveal that, when the projectile with similar characteristics $\left(V_{0}=1 \mathrm{~m} / \mathrm{s}, J_{0}=0.0243,0.0440,0.0645 \mathrm{Kgm}^{2}\right)$ impacts the beam at $X i=\frac{1}{5} L$, it slides and moves 
toward the tip of the beam and then gets back to the initial impact position, and finally leaves the beam. The higher moment of inertia, the longer impact time, the more maximum impact force and displacement. Comparing the results in Figs. 25 and 26 shows that the effect of inertia moment on the impact time becomes vise-versa when the projectile impacts at $X i=\frac{1}{5} L$.

In conjunction with results presented in Figs. 27 and 28, it can be found that the faster projectile with $V_{0}=2.5 \mathrm{~m} / \mathrm{s}$ and different moment of inertia passes the beam independent of impact location. Fig. 27 reveals an interesting point that, while the projectile with higher moment of inertia impacted at $X i=0$ induces large impact force, the displacement and impact time are similar for cases of high, moderate and low moment of inertia. However, when it impacts the beam at $X i=\frac{1}{5} L$ , the higher value of moment of inertia leads to shorter impact time but maximum displacement. Comparing the results in Figs. 26 and 28 also show that, for the case of impact on $X i=\frac{1}{5} L$, the increase in speed and energy of the projectile lead it pass the beam.

\section{Conclusions}

In this paper, the contact and impact behaviors of SMPs fabricated by 4D printing technology were explored via constitutive modeling, FEM formulation and simulation, as well as experiments. A novel phenomenological 3D constitutive model was derived to predict SME and visco-elasticplastic behaviors of SMPs in the large deformation regime. Non-linear 1D and 2D FEM governing equations were developed for the SMP beam in the plane stress condition under contact and impact loadings. Newmark numerical integration scheme coupled with Newton-Raphson iteration technique were implemented to solve non-linear governing equations in spatial and time fields 
following the visco-elastic-predictor visco-plastic-corrector return map scheme. The iterative node-to-surface algorithm was also imposed for global/local searching of the contact. The 3D FDM-based printer was used to print tensile samples as well as beams from polyurethane-based filaments. Thermo-mechanical experimental tests were first conducted to calibrate parameters introduced into the SMP constitutive model. Numerical studies were performed for the case of a cylinder in contact with an elastic-plastic substrate. Effects of substrate thickness, indentation location and edge effect, as well as validity of the Hertz theory for load-displacement response of elastic materials were examined. Afterwards, a set of numerical and experimental parametric study was directed to provide an insight into the influences of impact position and impactor initial velocity on the forced and free vibrational responses of the 4D printed SMP beams. Finally, the following main results can be concluded:

1) The results revealed that the constitutive model is able to well replicate elastic-plastic and hyperelastic behaviors of SMPs at low and high temperatures.

2) It was found that, for thin elastic substrates, the contact stiffness mostly remains unchanged in the middle of the substrate, while it drops down drastically in the edge area. However, this variation becomes smooth and gradually happens as the substrate thickness is increased. It was concluded that the Hertz theory is not valid to be used in the edge area.

3) It was found that $1 \mathrm{D}$ and $2 \mathrm{D}$ model results for the maximum and residual displacement of the beam, impactor velocity and forced-vibration configuration of the beam agree well with those from the experimental testing for low-velocity impact cases.

4) It was concluded that 2D FEM model replicates maximum displacement and impactor velocity more accurately compared to the $1 \mathrm{D}$ one when the impactor initial velocity is high.

5) It was seen that the high-velocity impact does not produce any plastic deformation.

6) The results showed that the large residual plastic deformation can be fully recovered by simply heating. 


\section{Acknowledgments}

Due to the absence of similar results in the specialized literature, this paper is likely to fill a gap in the state of the art of this problem, and provide pertinent results that are instrumental in the design of SMP beam-like structures under impact loadings.

The work described in this paper was supported by the Research Grants Council of the Hong Kong Special Administrative Region, China (Project No. CUHK 14202016) and The Chinese University of Hong Kong (Project ID: 3132823).

\section{References}

ASTM International, ASTM D638-10, 2010. Standard Test Method for Tensile Properties of Plastics 1-16.

Alwan, H.M. and Hamza, M.N., 2010. Hyperelastic constitutive modeling of rubber and rubberlike materials under finite strain. Engineering and Technology Journal, 28(13), pp.2560-2575.

Akbari, S., Sakhaei, A.H., Kowsari, K., Yang, B., Serjouei, A., Yuanfang, Z. and Ge, Q., 2018. Enhanced multimaterial 4D printing with active hinges. Smart Mater Struct 27(6), p.065027.

Boatti, E., Scalet, G., Auricchio, F., 2016. A three-dimensional finite-strain phenomenological model for shape-memory polymers: Formulation, numerical simulations, and comparison with experimental data. Int J Plast 83, 153-177.

Bodaghi, M., Damanpack, A.R., Liao, W.H., 2018. Triple shape memory polymers by 4D printing. Smart Mater Struct 27(6), p.065010.

Bodaghi, M. and Liao, W.H., 2019. 4D printed tunable mechanical metamaterials with shape memory operations. Smart Mater Struct 28(4), p.045019.

Christoforou, A.P., Yigit, A.S., 1998. Effect of flexibility on low velocity impact response. J Sound Vib 217, 563-578.

Chen, Y., Hou, S., Fu, K., Han, X., Ye, L., 2017. Low-velocity impact response of composite sandwich structures: Modelling and experiment. Compos Struct 168, 322-334.

Dintwa, E., Tijskens, E., Ramon, H., 2008. On the accuracy of the Hertz model to describe the normal contact of soft elastic spheres. Granul Matter 10, 209-221. 
Dunatunga, S., Kamrin, K., 2017. Continuum modeling of projectile impact and penetration in dry granular media. J Mech Phys Solids 100, 45-60.

Fan, X., Chung, J.Y., Lim, Y.X., Li, Z., Loh, X.J., 2016. Review of adaptive programmable materials and their bioapplications. ACS app Mater Interface 8(49), 33351-33370.

Fan, Y., Xiang, Y., Shen, H.S., Wang, H., 2018. Low-velocity impact response of FG-GRC laminated beams resting on visco-elastic foundations. Int J Mech Sci 141, 117-126,

Ge, Q., Dunn, C.K., Qi, H.J. and Dunn, M.L., 2014. Active origami by 4D printing. Smart Mater Struct 23(9), p.094007.

Guo, X., Liu, L., Zhou, B., Liu, Y., Leng, J., 2015. Influence of strain rates on the mechanical behaviors of shape memory polymer. Smart Mater Struct 24(9), p.095009.

Hertz, H., 1986. Über die Berührung fester elastischer Körper. In: Jones DE, editor. On the contact of elastic solids. London: Macmillan.

Hills, D., Nowell, D., Sackfield, A., 1993. Mechanics of elastic contacts. Oxford: ButterworthHeineman Ltd.

Ivañez, I., Barbero, E., Sanchez-Saez, S., 2014. Analytical study of the low-velocity impact response of composite sandwich beams. Compos Struct 111, 459-467.

Liu, Y., Du, H., Liu, L., Leng, J., 2014. Shape memory polymers and their composites in aerospace applications: a review. Smart Mater Struct 23(2), p.023001.

Lu, H., Wang, X., Yu, K., Fu, Y.Q.R. and Leng, J., 2019. A thermodynamic model for tunable multi-shape memory effect and cooperative relaxation in amorphous polymers. Smart Mater Struct 28 p.025031.

Lin, C., Lv, J., Li, Y., Zhang, F., Li, J., Liu, Y., Liu, L. and Leng, J., 2019. 4D-printed biodegradable and remotely controllable shape memory occlusion devices. Adv Function Mater 29(51), p.1906569.

Liu, T., Liu, L., Zeng, C., Liu, Y. and Leng, J., 2020. 4D printed anisotropic structures with tailored mechanical behaviors and shape memory effects. Compos Sci Tech 186, p.107935.

Mao, Y., Robertson, J.M., Mu, X., Mather, P.T., Qi, H.J., 2015. Thermoviscoplastic behaviors of anisotropic shape memory elastomeric composites for cold programmed non-affine shape change. J Mech Phys Solids 85, 219-244.

Pieczyska, E.A., Staszczak, M., Maj, M., Kowalczyk-Gajewska, K., Golasiński, K., Cristea, M., Tobushi, H., Hayashi, S., 2016. Investigation of thermomechanical couplings, strain localization and shape memory properties in a shape memory polymer subjected to loading at various strain rates. Smart Mater Struct 25(8), p.085002.

Park, H., Harrison, P., Guo, Z., Lee, M.G. and Yu, W.R., 2016. Three-dimensional constitutive model for shape memory polymers using multiplicative decomposition of the deformation gradient and shape memory strains. Mech Material, 93, pp.43-62.

Reddy, J.N., 2004. An introduction to nonlinear finite element analysis. New York: Oxford University Press.

Ranjbar, M., Feli, S., 2018. Low velocity impact analysis of an axially functionally graded carbon nanotube reinforced cantilever beam. Polymer Compos 39, E969-E983. 
Simo, J.C., Hughes, T.J.R., 1998. Computational inelasticity. New York: Springer.

Wang, H., Yin. X., Deng, Q., Yu, B., Hao, Q., Dong, X., 2017. Experimental and theoretical analyses of elastic-plastic repeated impacts by considering wave effects. Euro J Mech-A/Solids 65: 212-222.

Zhang, L., Yin, X., Yang, J., Wang, H., Deng, Q., Yu, B., Hao, Q., Ding, H., Qi, X., Jin, T., Dong, X., 2018. Transient impact response analysis of an elastic-plastic beam. App Math Model 55, 616-636.

Zeng, H., Xie, Z., Gu, J., Sun, H., 2018. A 1D thermomechanical network transition constitutive model coupled with multiple structural relaxation for shape memory polymers. Smart Mater Struct 27(3), p.035024.

Zeng, H., Liu, J., Xie, Z. and Sun, H., 2019a. Modeling the shape memory and strength properties of fiber-reinforced shape memory polymer composite laminates. Smart Mater Struct 28(10), p.105011.

Zeng, H., Leng, J., Gu, J. and Sun, H., 2019b. Modeling the thermomechanical behaviors of shape memory polymers and their nanocomposites by a network transition theory. Smart Mater Struct, 28(6), p.065018.

Zeng, H., Pan, N., Gu, J. and Sun, H., 2020. Modeling the thermoviscoelasticity of transversely isotropic shape memory polymer composites. Smart Mater Struct 29(2), p.025012.

Zienkiewicz, O.C., Taylor, R.L., Fox, D.A., 2014. The finite element method for solid and structural mechanics. Elsevier Ltd. 


\section{List of Figures:}

Fig. 1. Schematic diagram of the rigid indentation.

Fig. 2. Contact zone and definition of normal and tangential directions.

Fig. 3. Beam element (a) under deformation (b).

Fig. 4. Three-nodded linear triangular element.

Fig. 5. Three-nodded Hermitian triangular element.

Fig. 6. Schematic diagram of the pendulum impact test system and the beam.

Fig. 7. Schematic diagram of the impacted beam.

Fig. 8. DMA test in terms of storage modulus and tan $(\delta)$.

Fig. 9. Experimental and numerical elastic modulus extracted from the DMA test.

Fig. 10. Stress-strain behaviors of the printed SMP at low (a) $23{ }^{\circ} \mathrm{C}$ and high (b) $90{ }^{\circ} \mathrm{C}$ temperatures.

Fig. 11. A substrate in contact with a cylindrical rigid indenter.

Fig. 12. Variation of contact stiffness versus different positions.

Fig. 13. Variation of contact stiffness versus different substrate thickness at the edge and middle positions.

Fig. 14. Variation of contact stiffness ratio versus different substrate thickness.

Fig. 15. Load-indentation curve at the edge and center of the SMP substrate.

Fig. 16. Variation of contact stiffness ratio along the length of the SMP substrate.

Fig. 17. Time history of displacement (a), impactor velocity (b), impact force (c), and energy (d) of the SMP beam impacted by $V_{0}=1 \mathrm{~m} / \mathrm{s}$ at $X i=0$.

Fig. 18. Configuration of the beam during the forced vibration range $\left(V_{0}=1 \mathrm{~m} / \mathrm{s}, X i=0\right)$. (color bar shows axial stress)

Fig. 19. Time history of displacement (a), impactor velocity (b), impact force (c), and energy (d) of the SMP beam impacted by $V_{0}=2 \mathrm{~m} / \mathrm{s}$ at $X i=0$.

Fig. 20. Configuration of the beam during the forced vibration range $\left(V_{0}=2 \mathrm{~m} / \mathrm{s}, X i=0\right)$. (color bar shows axial stress)

Fig. 21. Time history of displacement (a), impactor velocity (b) of the SMP beam impacted by $V_{0}=2.5 \mathrm{~m} / \mathrm{s}$ at $X i=0$. 
Fig. 22. Time history of displacement (a), impactor velocity (b) of the SMP beam impacted by $V_{0}=1 \mathrm{~m} / \mathrm{s}$ at $X i=\frac{1}{5} L$.

Fig. 26. Time history of displacement (a) and impact force (b) of the SMP beam impacted by $V_{0}=1 \mathrm{~m} / \mathrm{s}$ at $X i=\frac{1}{4} L$ with different moment of inertia.

Fig. 23. Time history of displacement (a), impactor velocity (c) of the SMP beam impacted by $V_{0}=2.5 \mathrm{~m} / \mathrm{s}$ at $X i=\frac{1}{5} \mathrm{~L}$. Shape recovery under temperature control (c).

Fig. 24. Configuration of the beam during the forced vibration range $\left(V_{0}=2.5 \mathrm{~m} / \mathrm{s}, X_{i}=\frac{1}{5} L\right)$. (color bar shows axial stress)

Fig. 25. Time history of displacement (a) and impact force (b) of the SMP beam impacted by $V_{0}=1 \mathrm{~m} / \mathrm{s}$ at $X i=0$ with different moment of inertia.

Fig. 27. Time history of displacement (a) and impact force (b) of the SMP beam impacted by $V_{0}=2.5 \mathrm{~m} / \mathrm{s}$ at $X i=0$ with different moment of inertia.

Fig. 28. Time history of displacement (a) and impact force (b) of the SMP beam impacted by $V_{0}=2.5 \mathrm{~m} / \mathrm{s}$ at $X i=\frac{1}{4} L$ with different moment of inertia. 


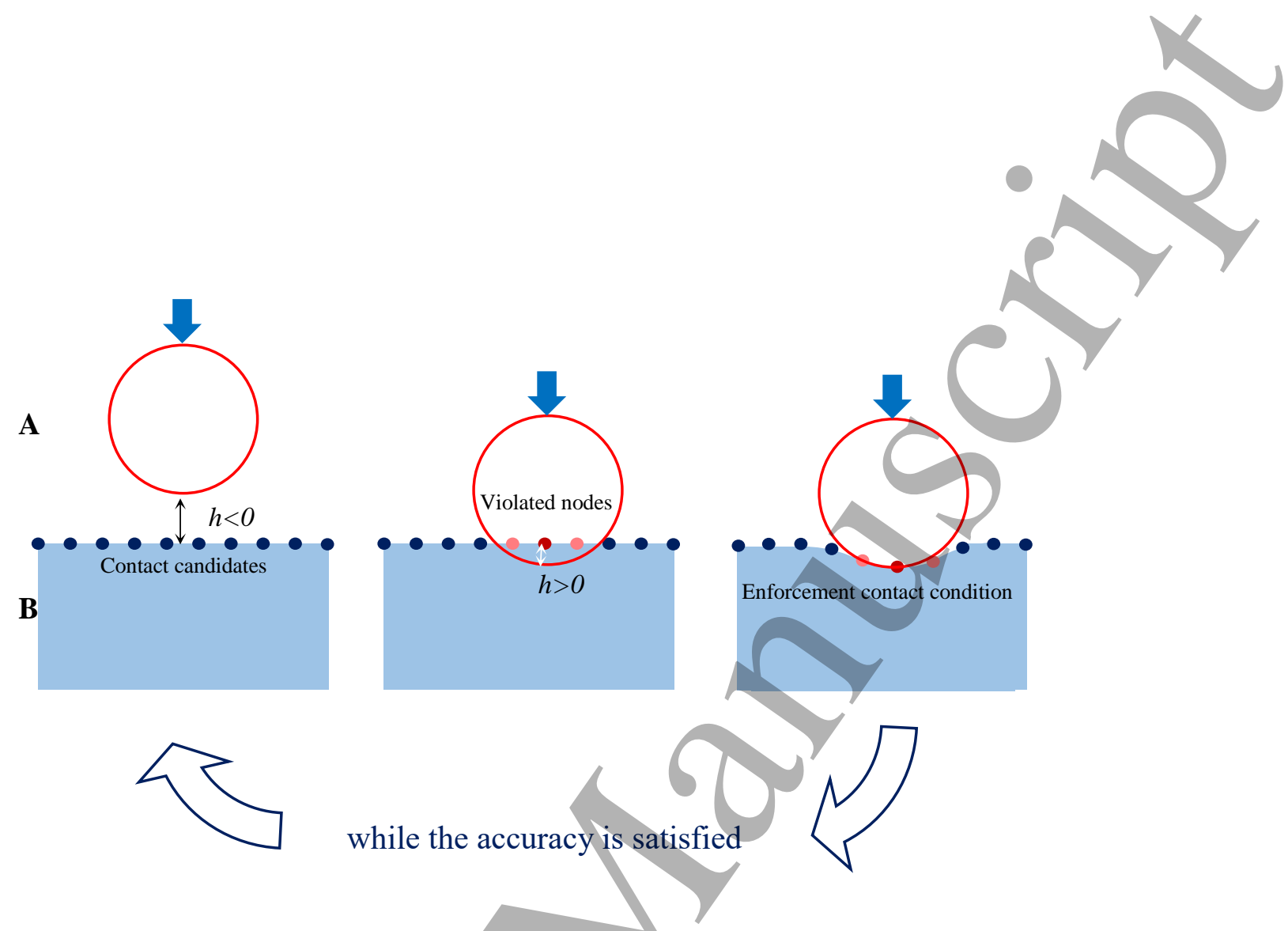

Fig. 1. Schematic diagram of the rigid indentation. 
Fig. 2. Contact zone and definition of normal and tangential directions. 


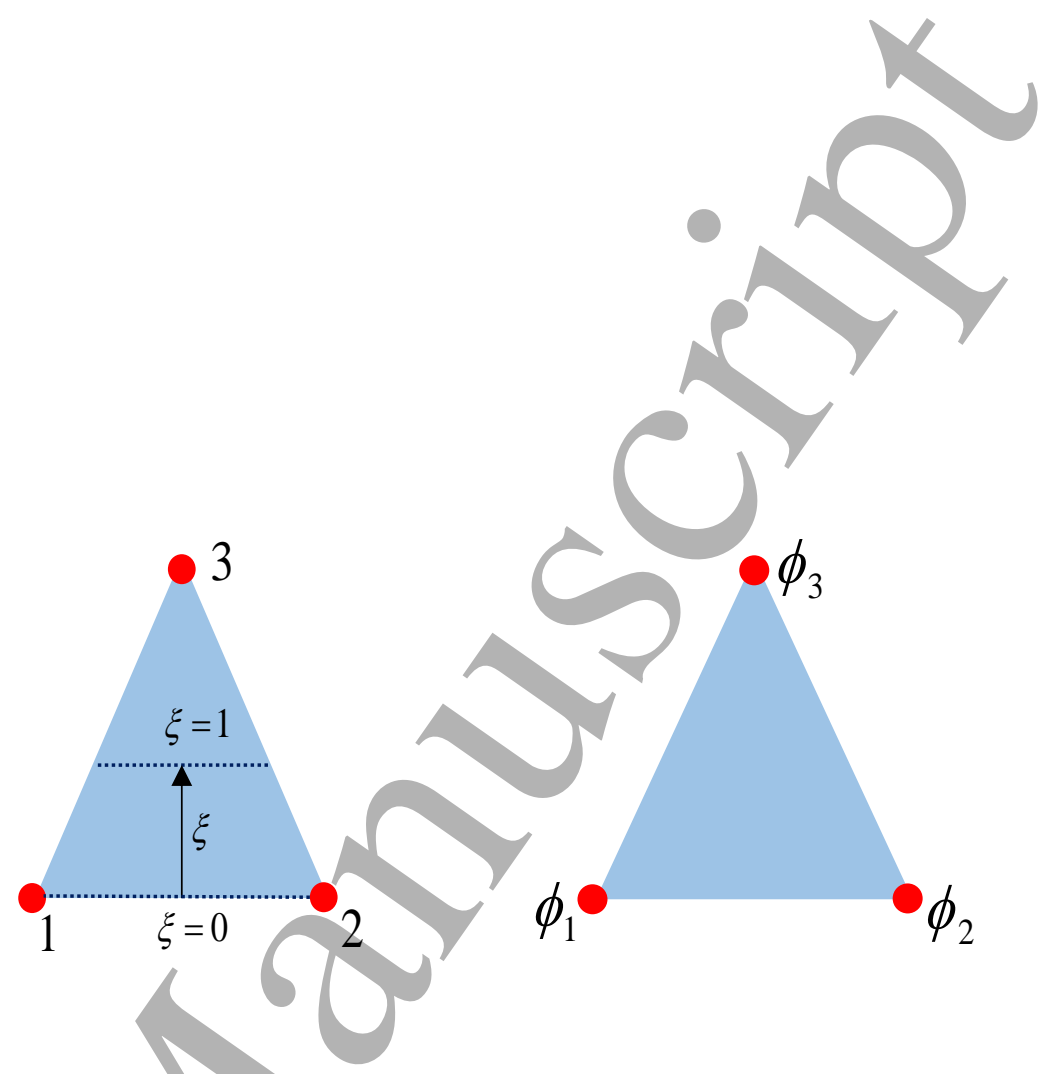

Fig. 4. Three-nodded linear triangular element.

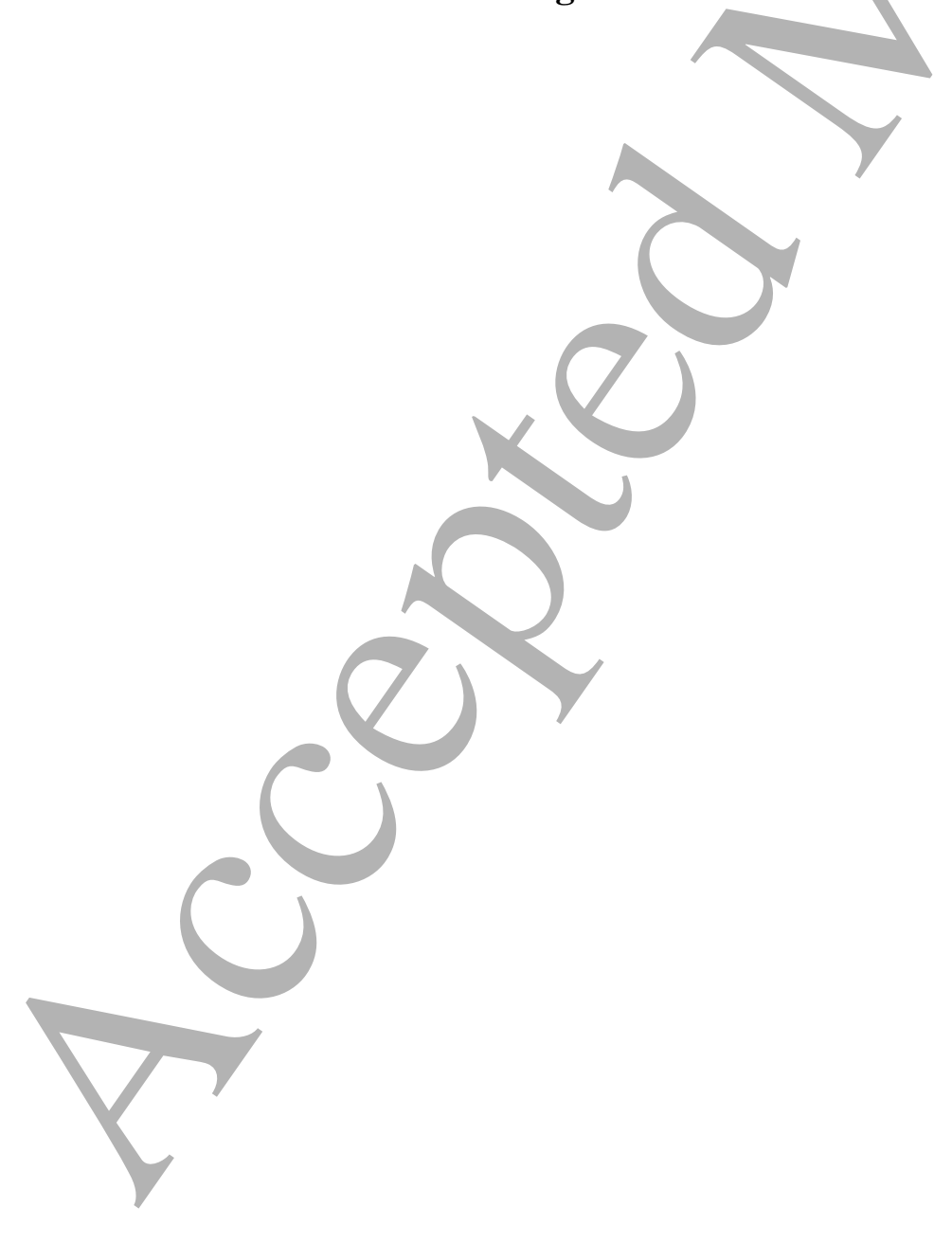


Fig. 5. Three-nodded Hermitian triangular element. 


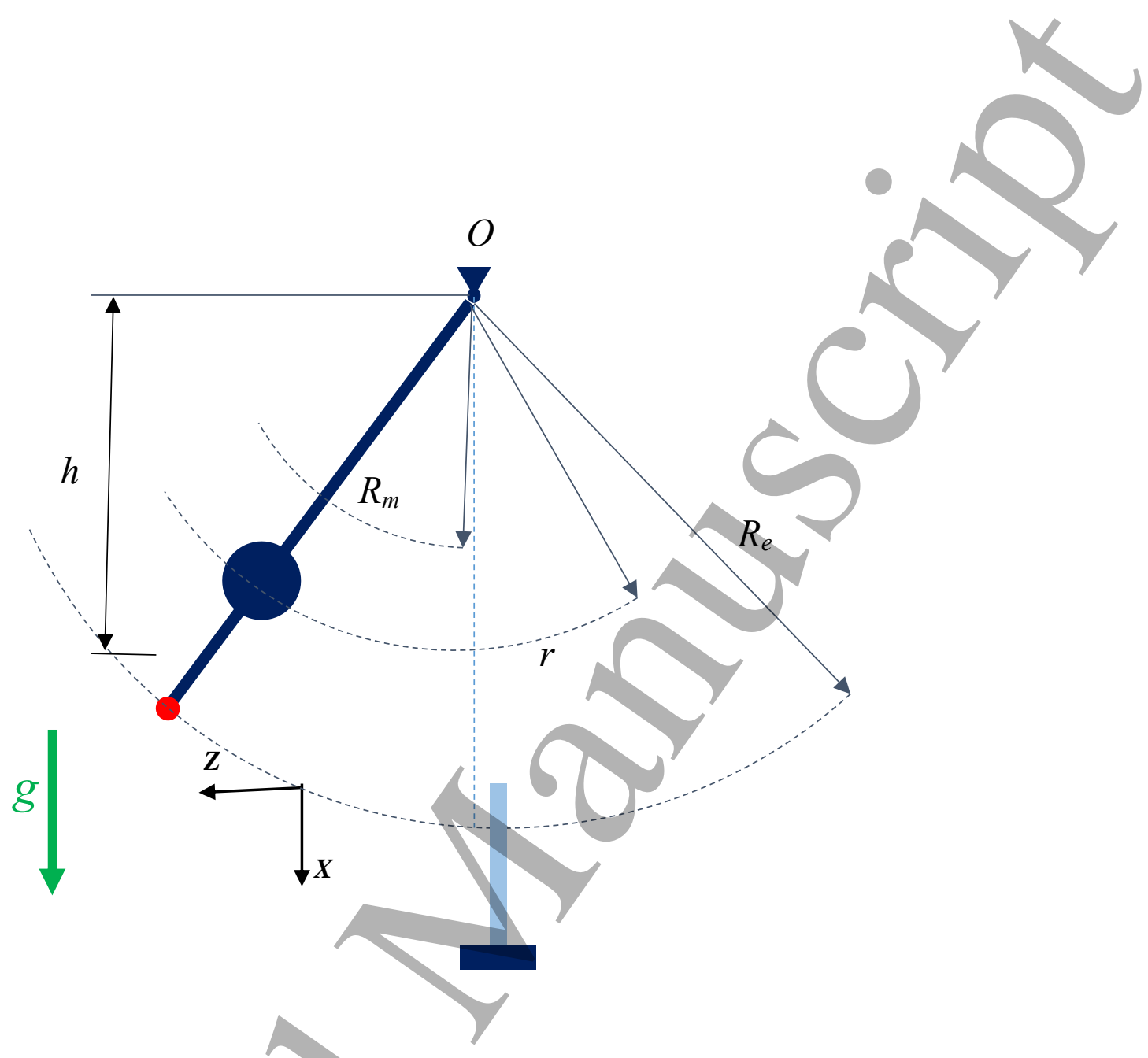

Fig. 6. Schematic diagram of the pendulum impact test system and the beam. 


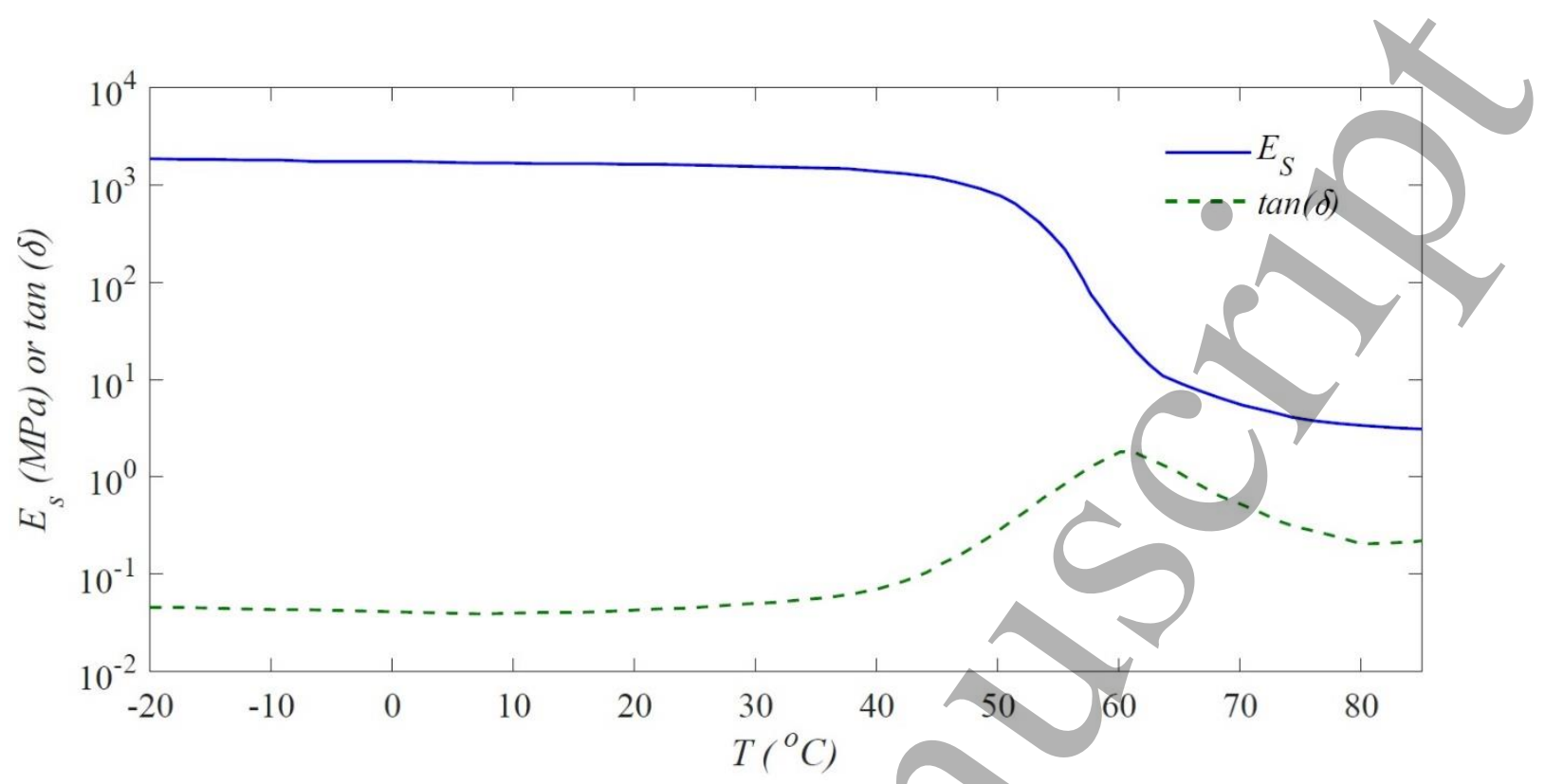

Fig. 8. DMA test in terms of storage modulus and $\tan (\delta)$. 


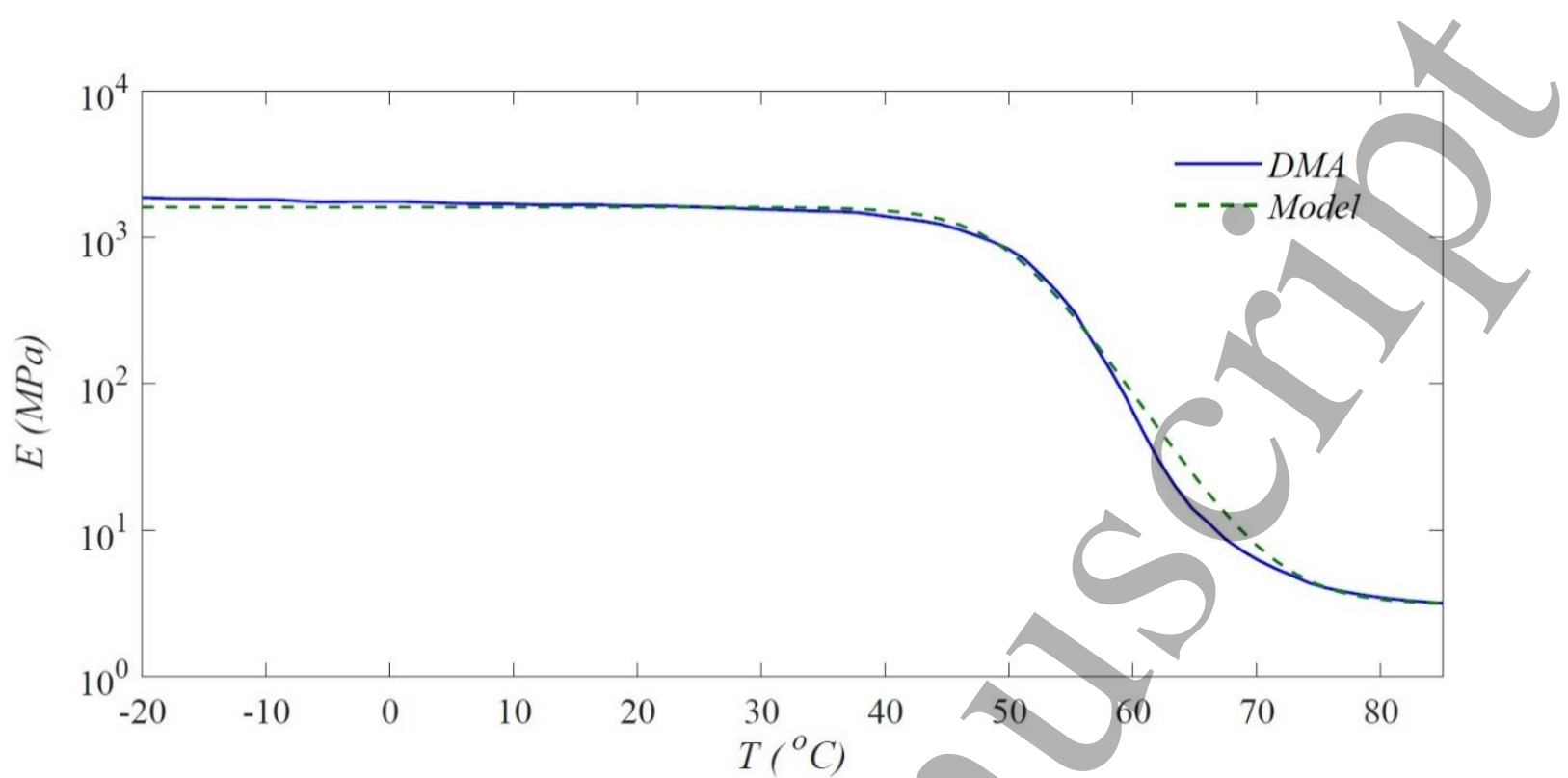

Fig. 9. Experimental and numerical elastic modulus extracted from the DMA test.

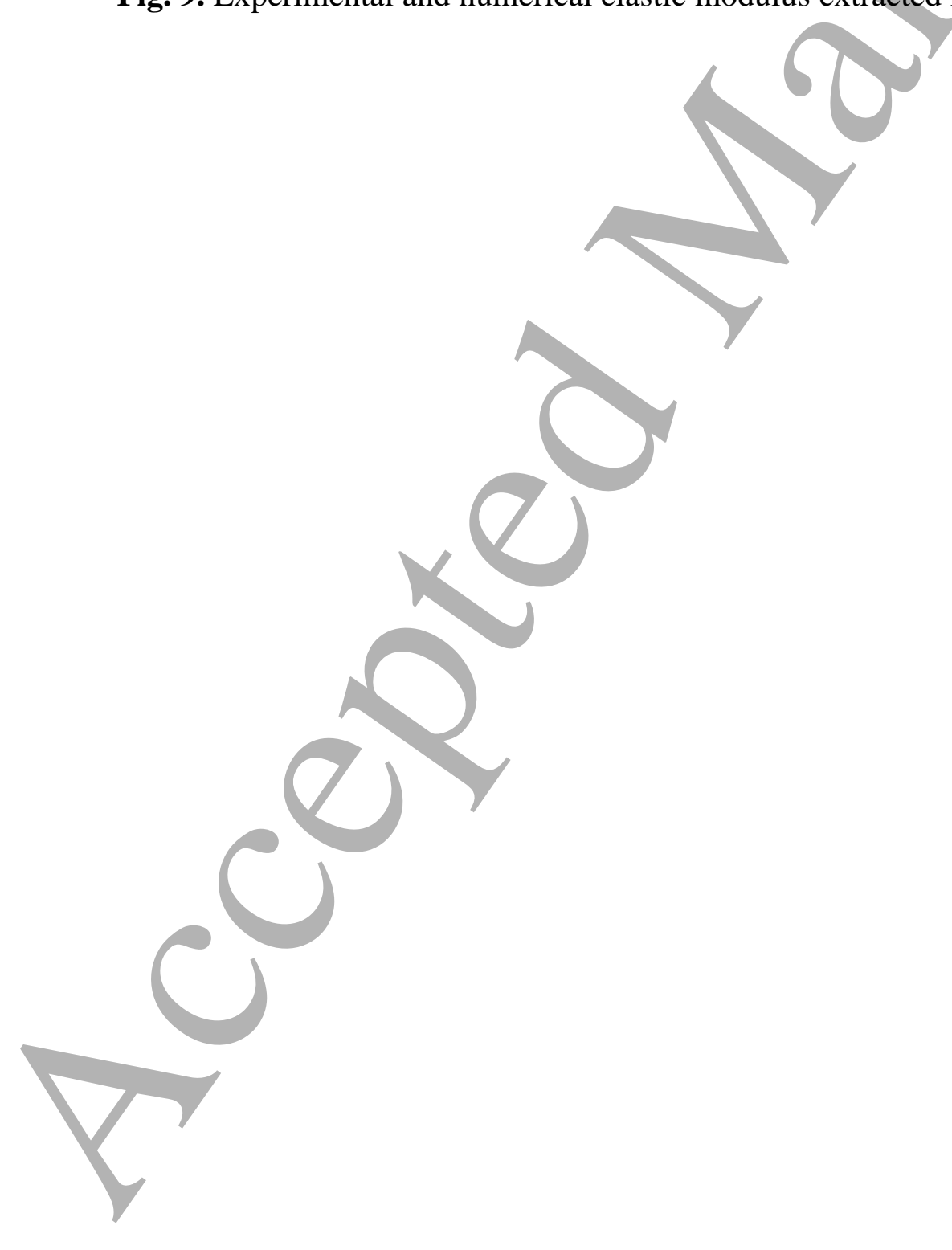


(a)
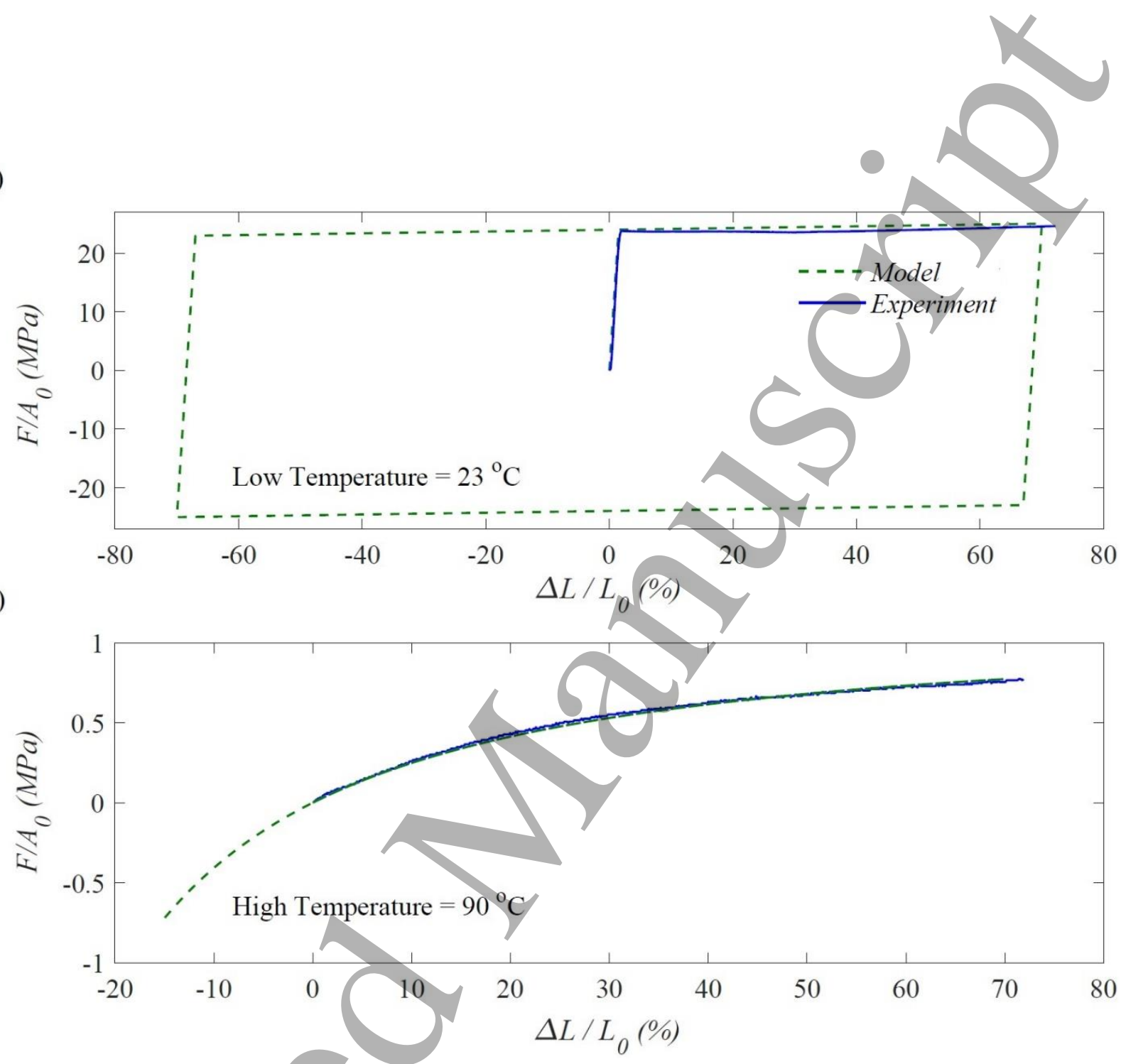

Fig. 10. Stress-strain behaviors of the printed SMP at low (a) $23{ }^{\circ} \mathrm{C}$ and high (b) $90{ }^{\circ} \mathrm{C}$ temperatures. 


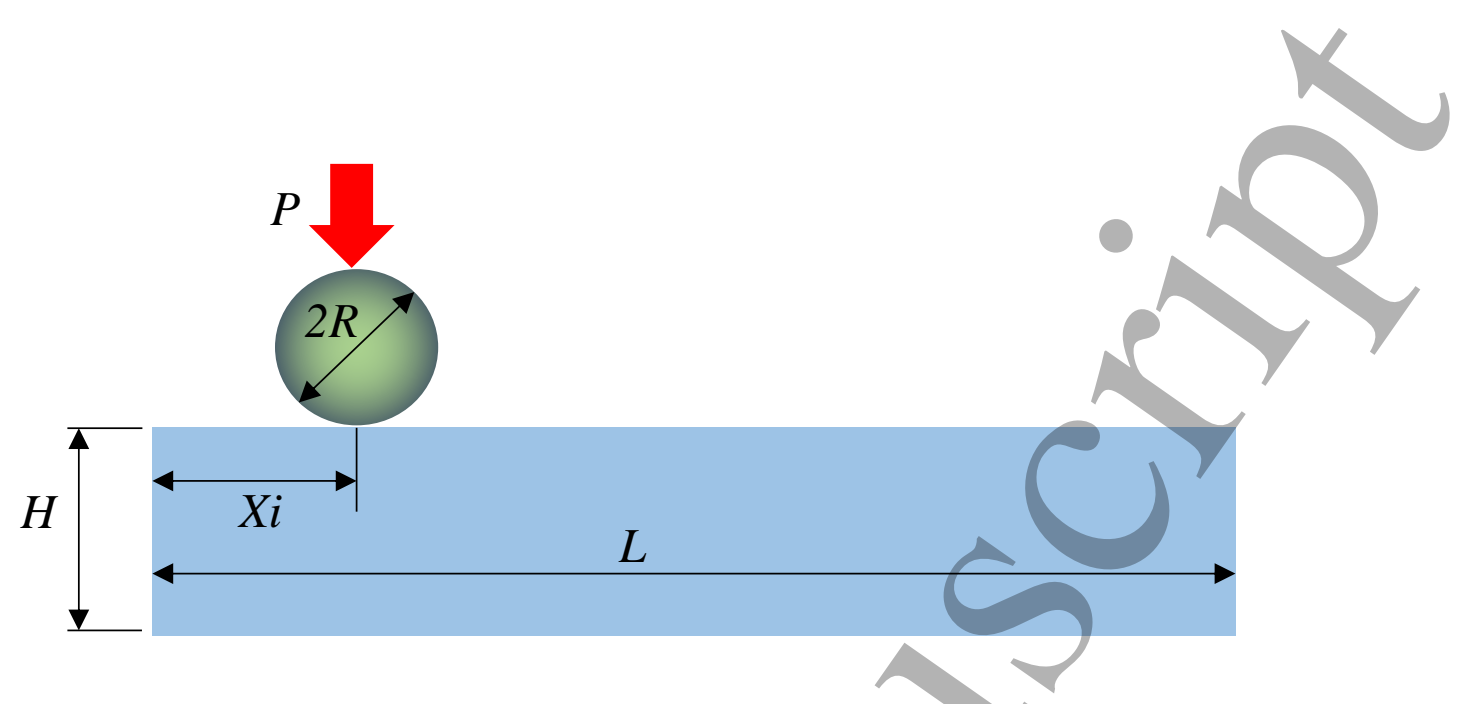

Fig. 11. A substrate in contact with a cylindrical rigid indenter. 


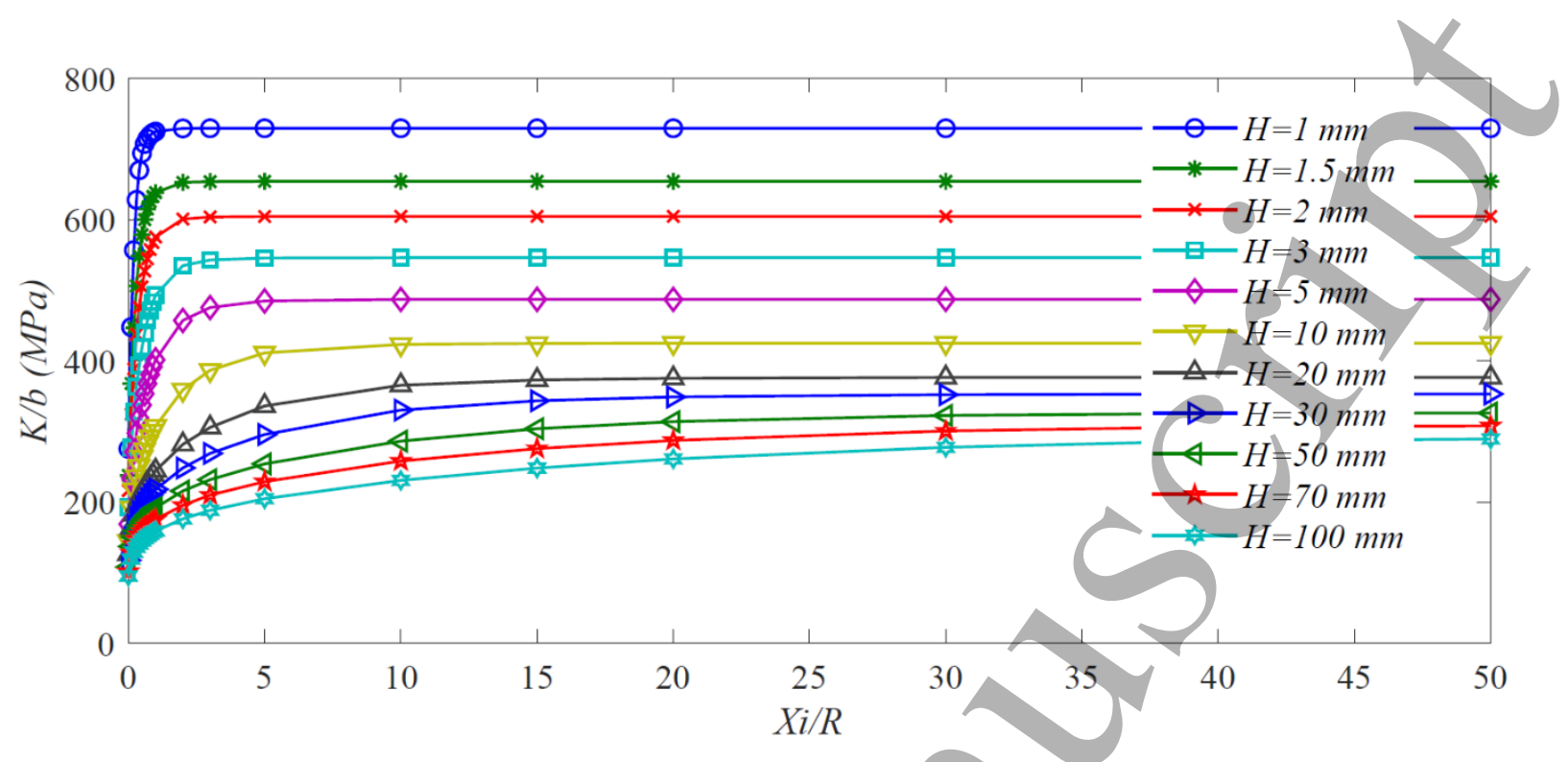

Fig. 12. Variation of contact stiffness versus different positions. 


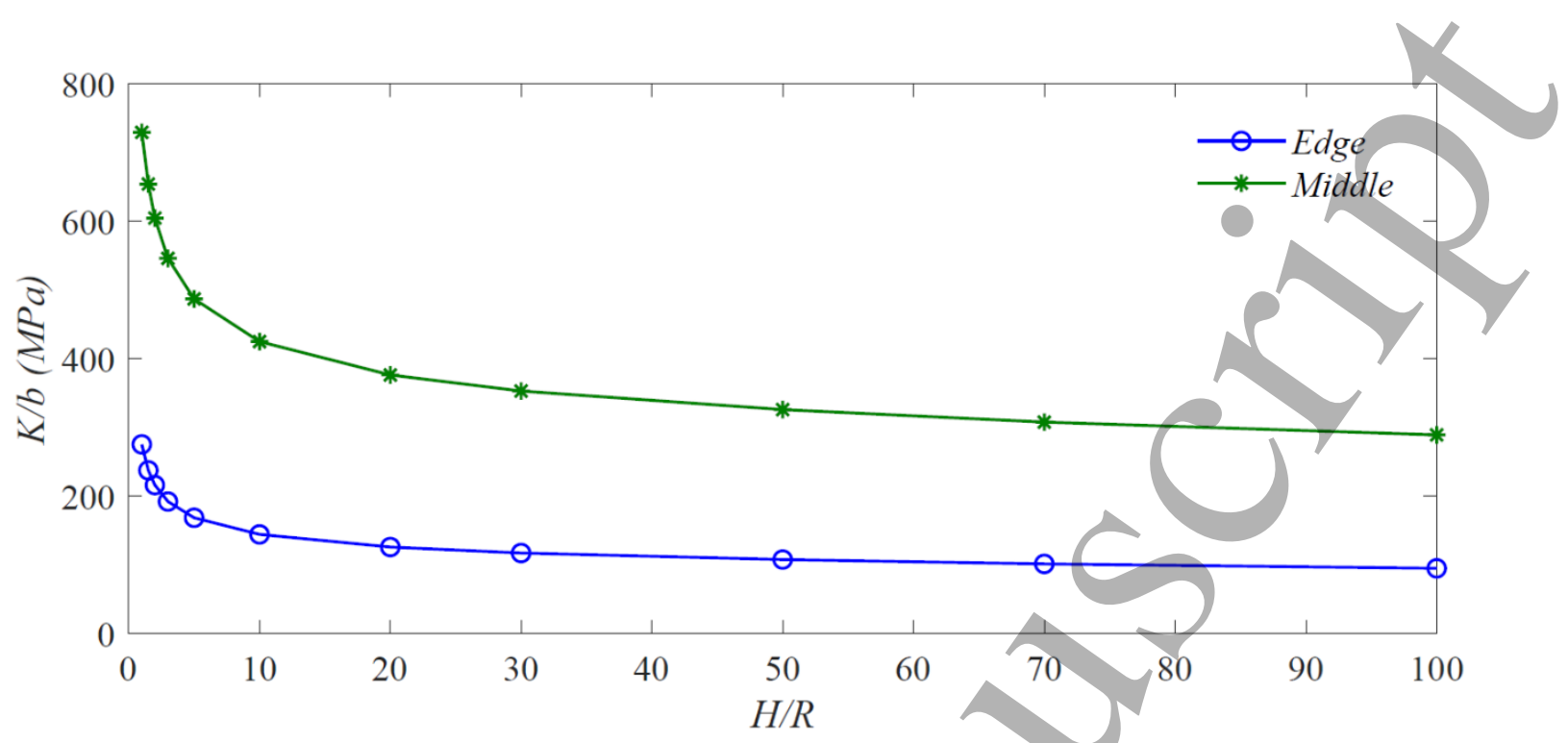

Fig. 13. Variation of contact stiffness versus different substrate thickness at the edge and middle positions. 


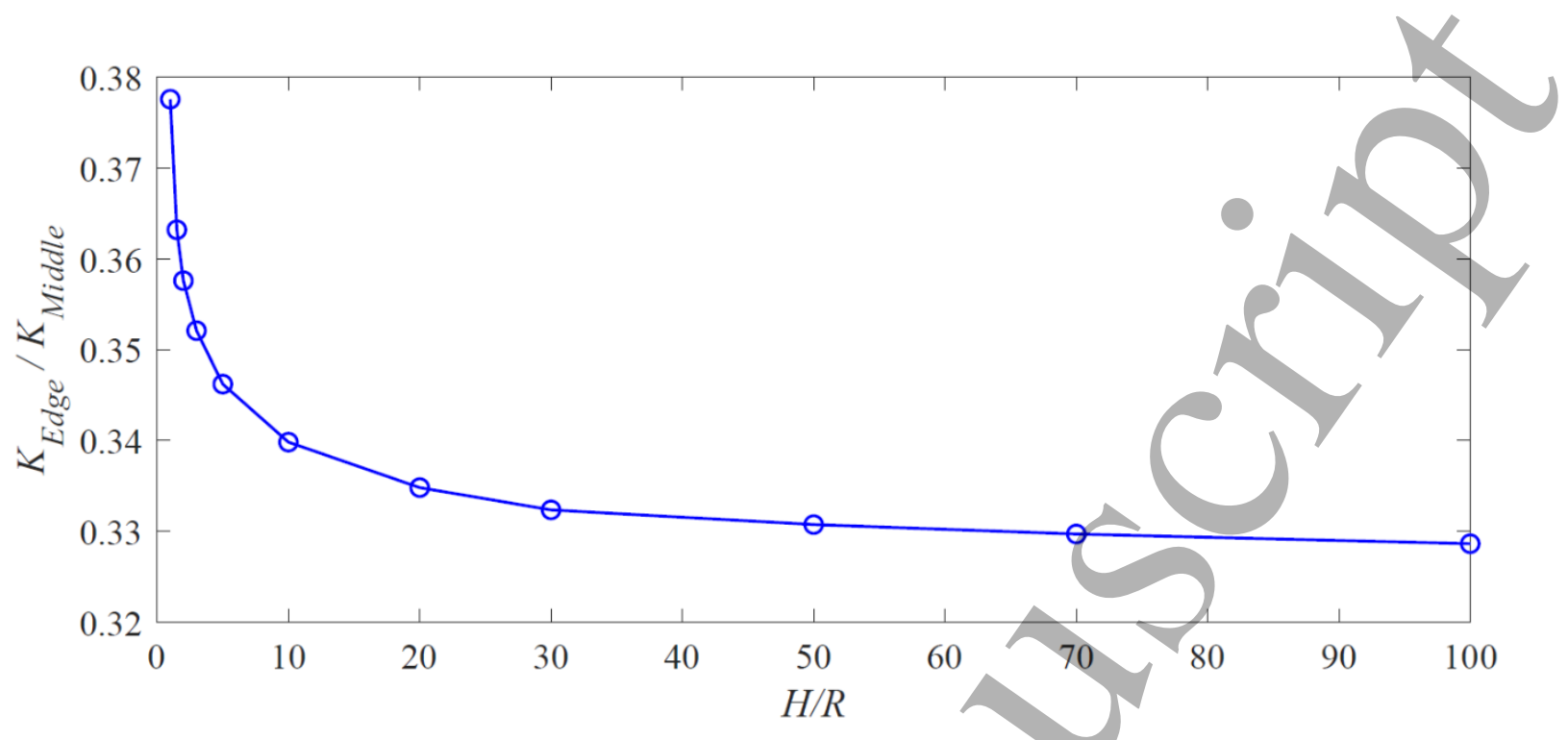

Fig. 14. Variation of contact stiffness ratio versus different substrate thickness.

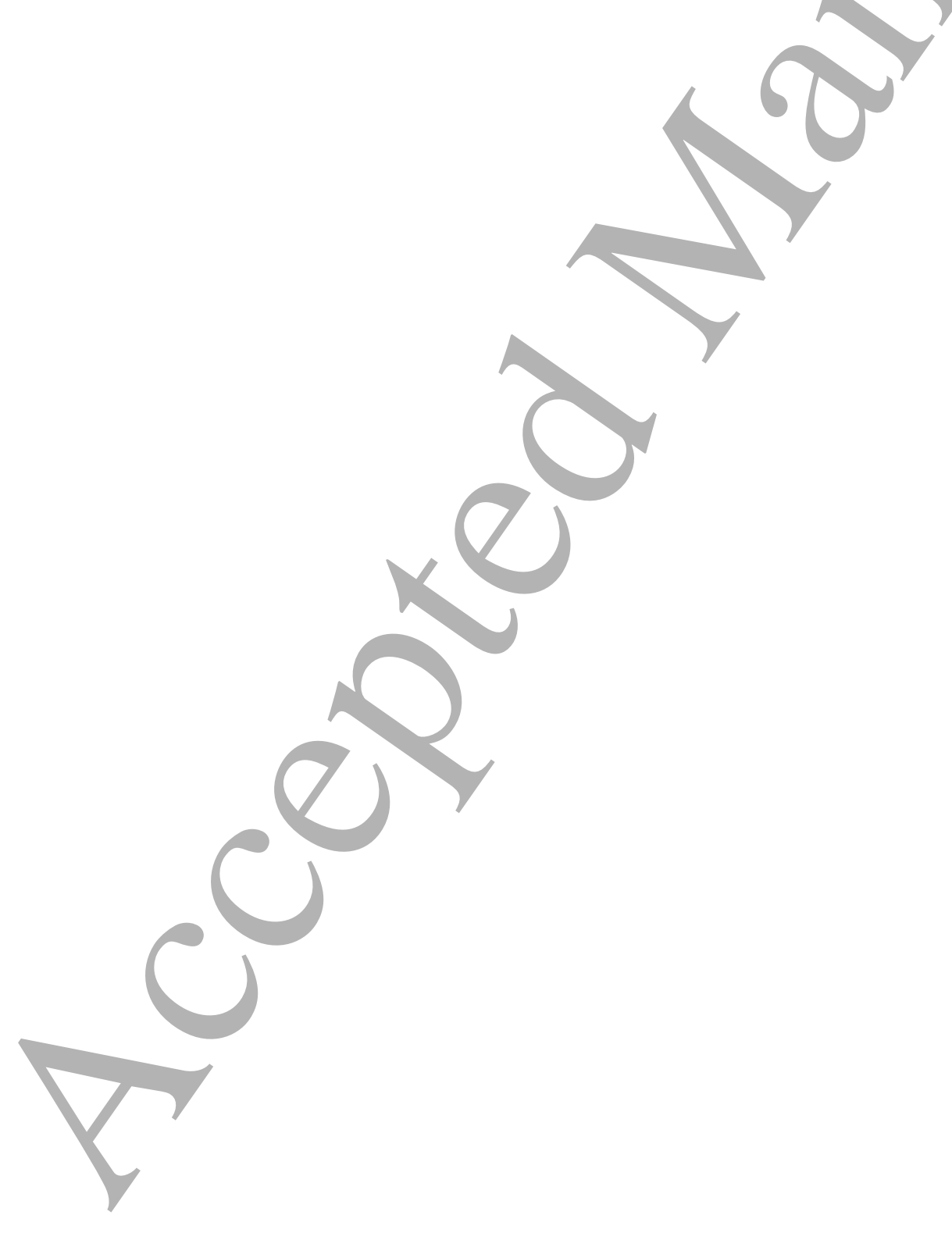




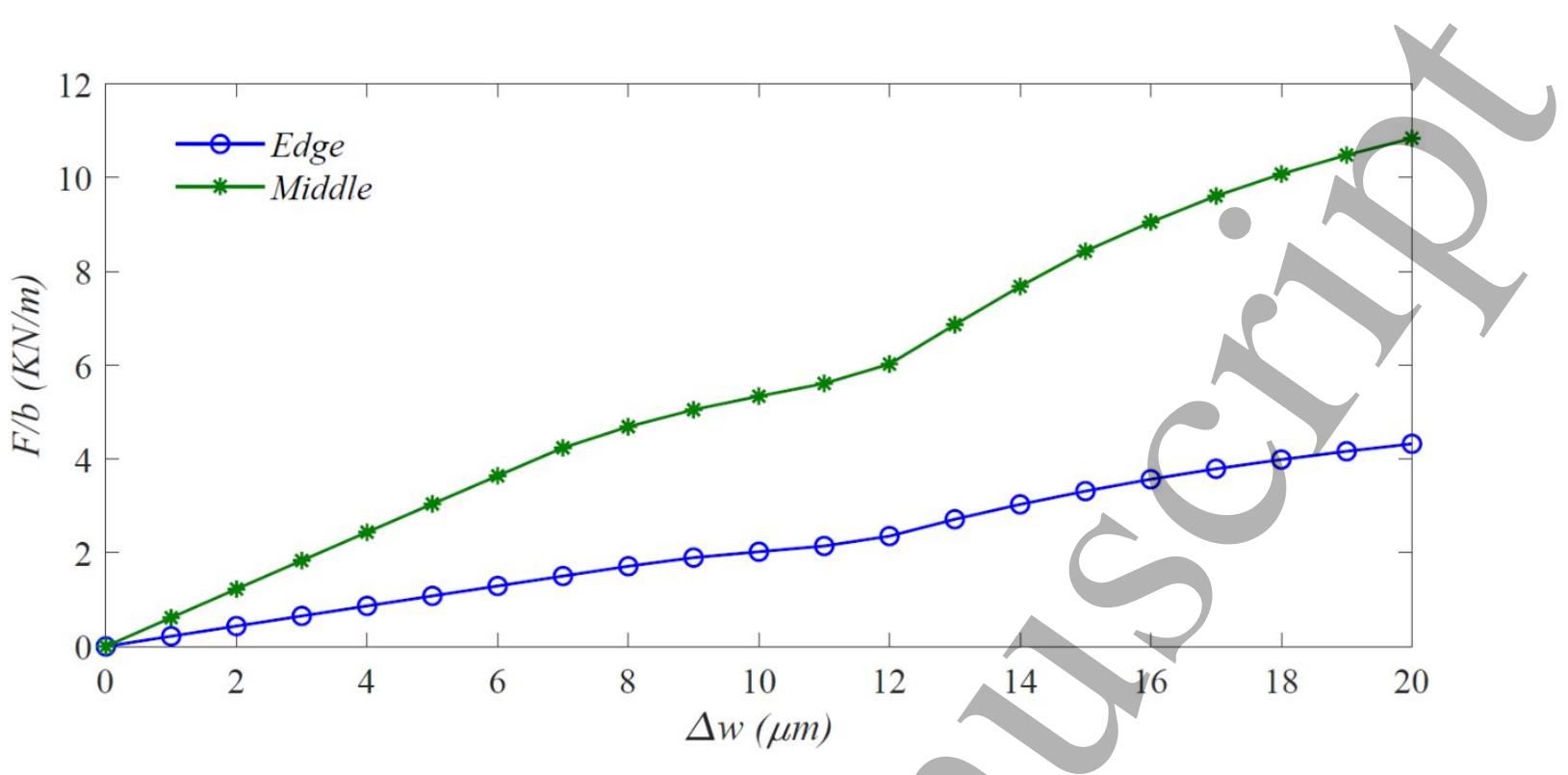

Fig. 15. Load-indentation curve at the edge and center of the SMP substrate. 


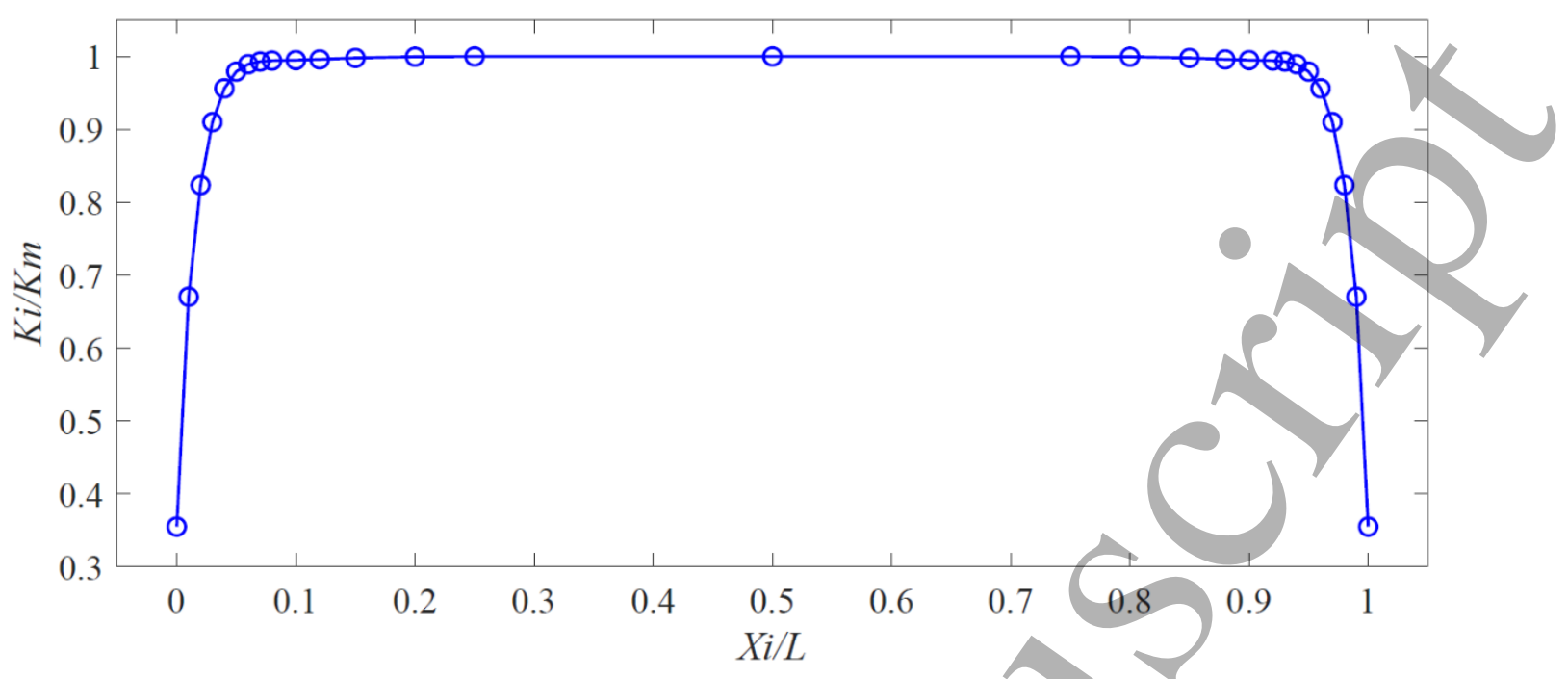

Fig. 16. Variation of contact stiffness ratio along the length of the SMP substrate.

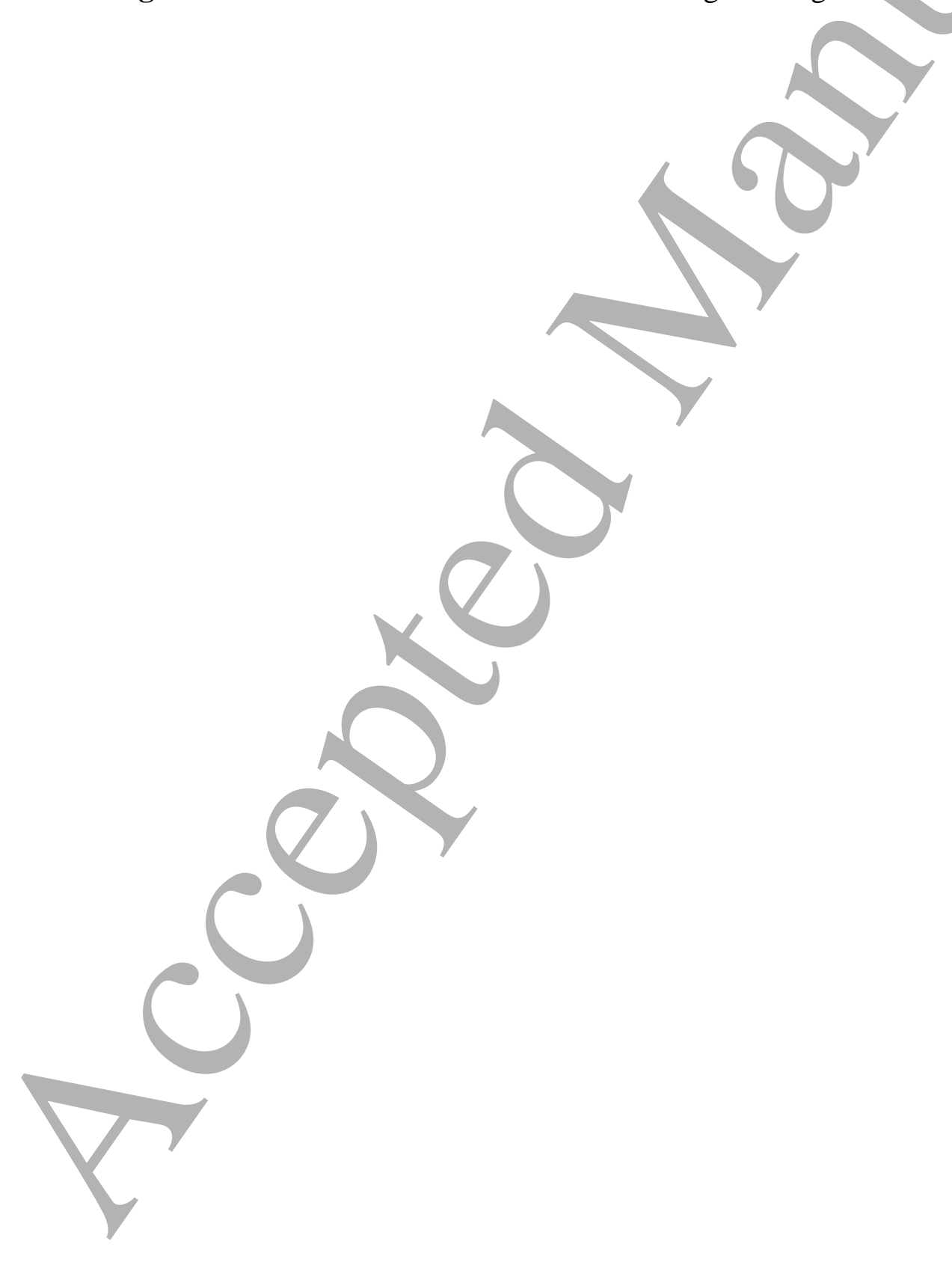


(a)

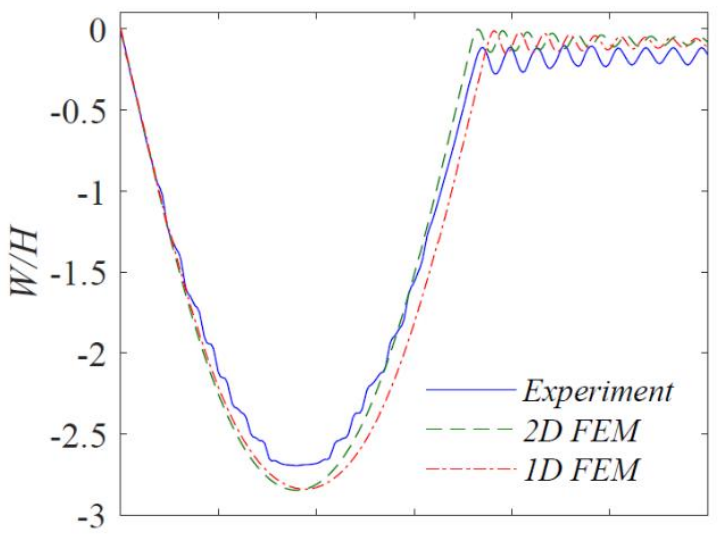

(c)

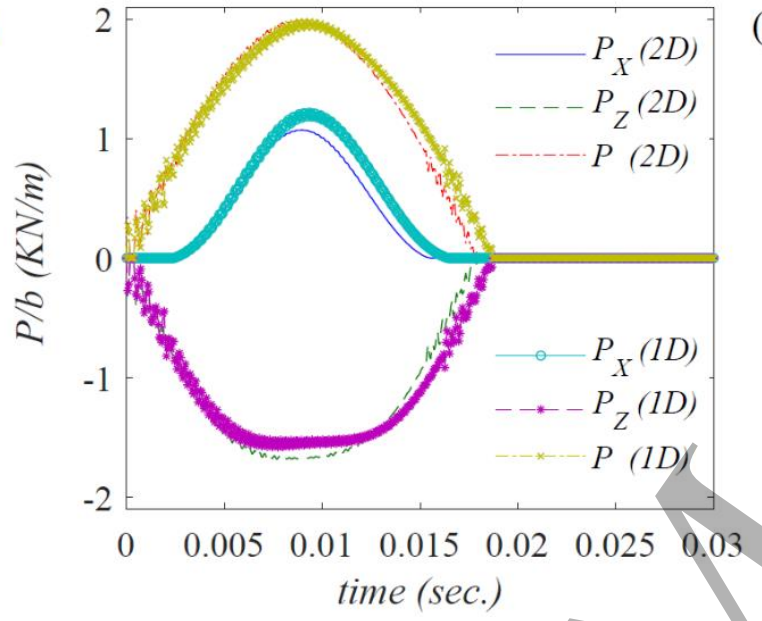

(b)

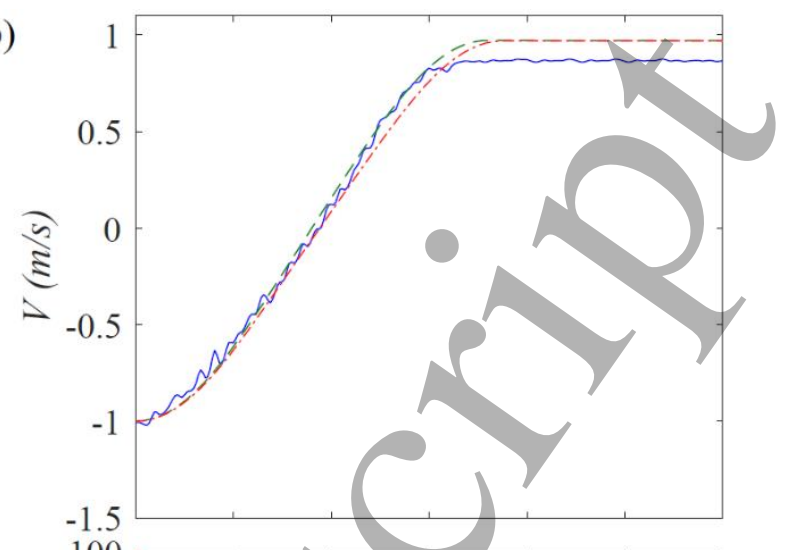

(d)

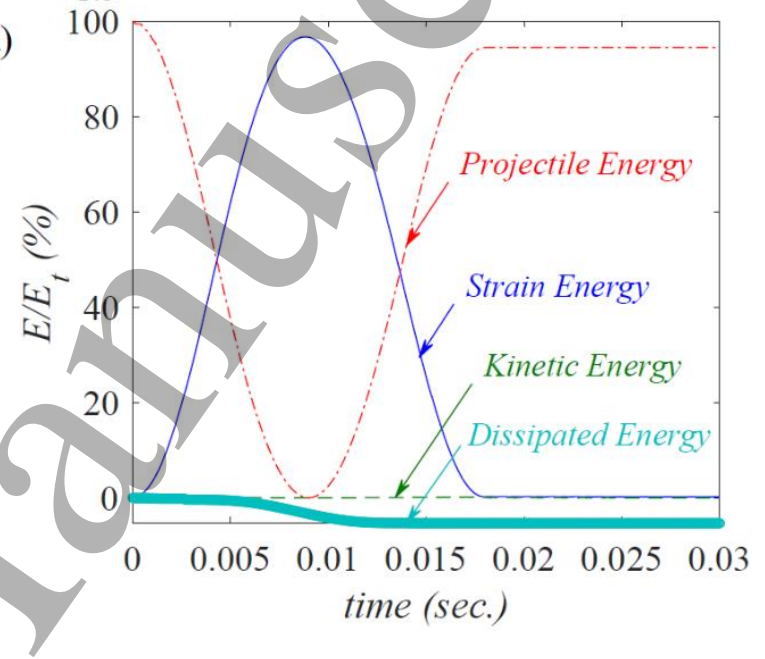

Fig. 17. Time history of displacement (a), impactor velocity (b), impact force (c), and energy (d) of the SMP beam impacted by $V_{0}=1 \mathrm{~m} / \mathrm{s}$ at $X i=0$. 

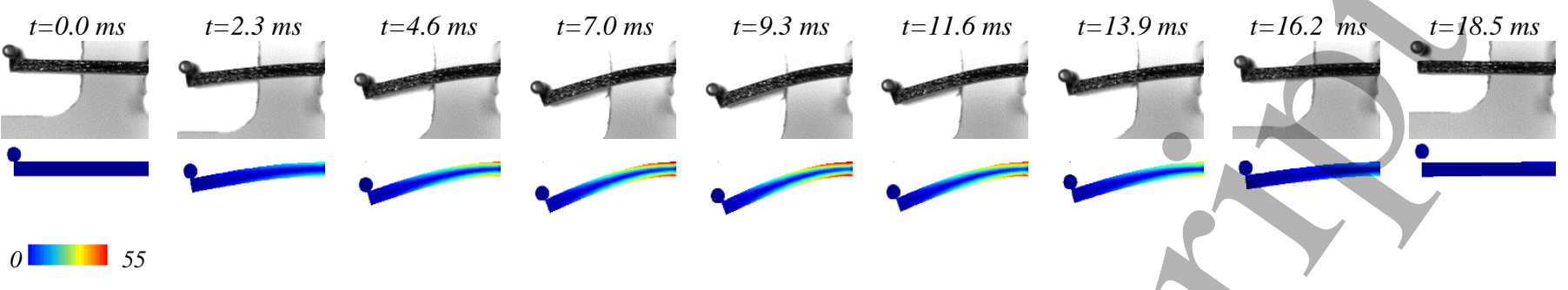

Fig. 18. Configuration of the beam during the forced vibration range $\left(V_{0}=1 \mathrm{~m} / \mathrm{s}, X i=0\right)$. (color bar shows axial stress)

1
15
16
17
18
19
20
2
2
23
2
25
2
2
28
2
30
3
32
33
3
35
36
3
38
39
40
4
42
43
4
45
46
4
48
4
50
5
52
53
5
55
56
58
59


(a)

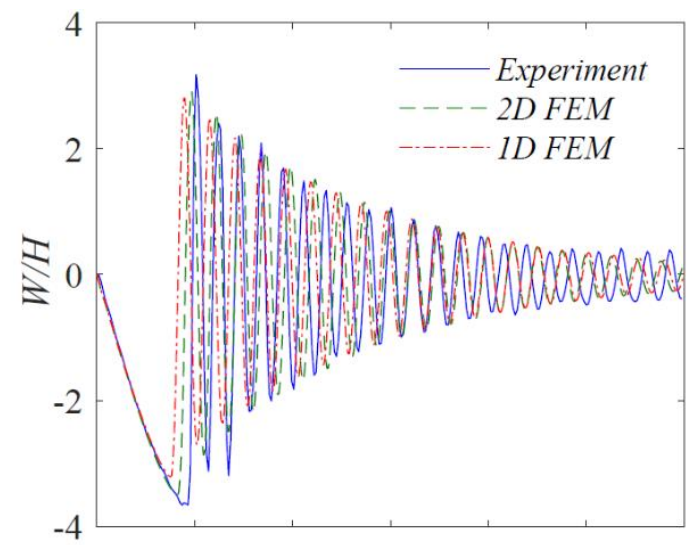

(c)

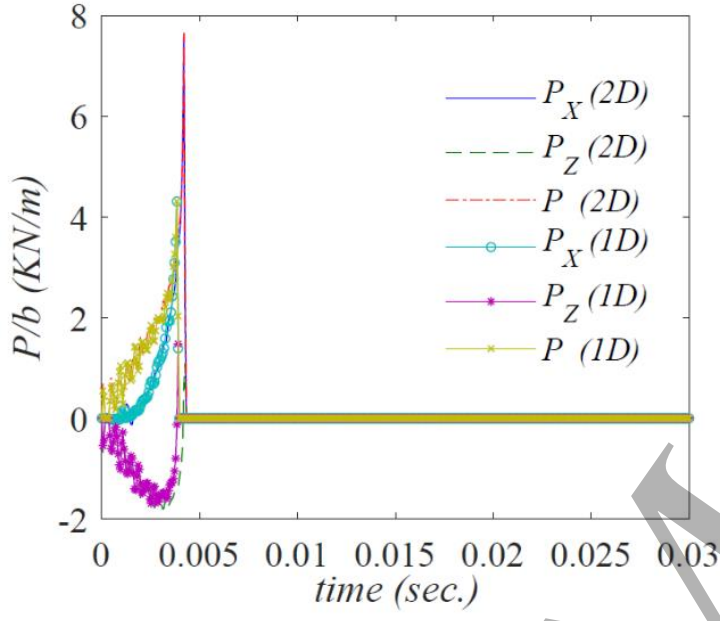

(b)

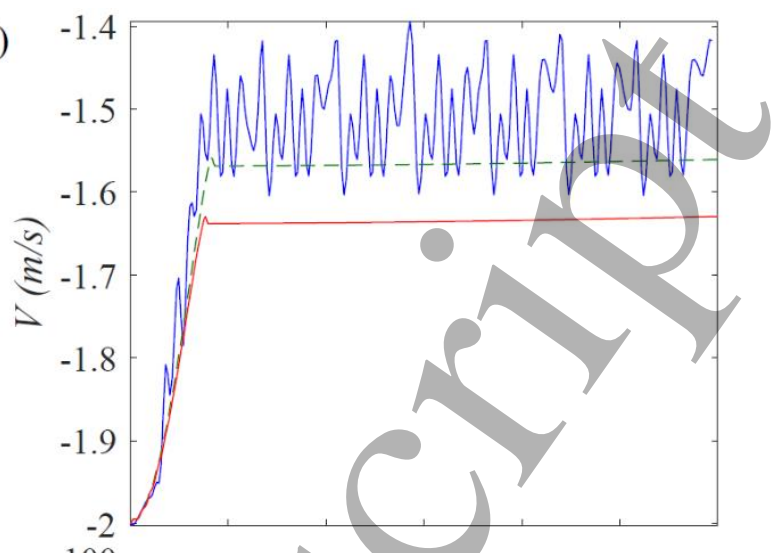

(d)

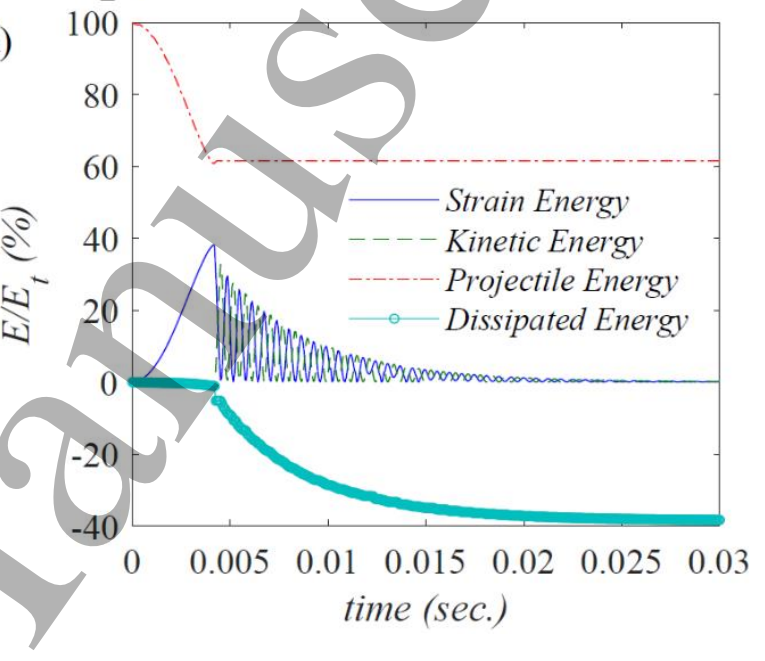

Fig. 19. Time history of displacement (a), impactor velocity (b), impact force (c), and energy (d) of the SMP beam impacted by $V_{0}=2 \mathrm{~m} / \mathrm{s}$ at $X i=0$. (color bar shows axial stress) 


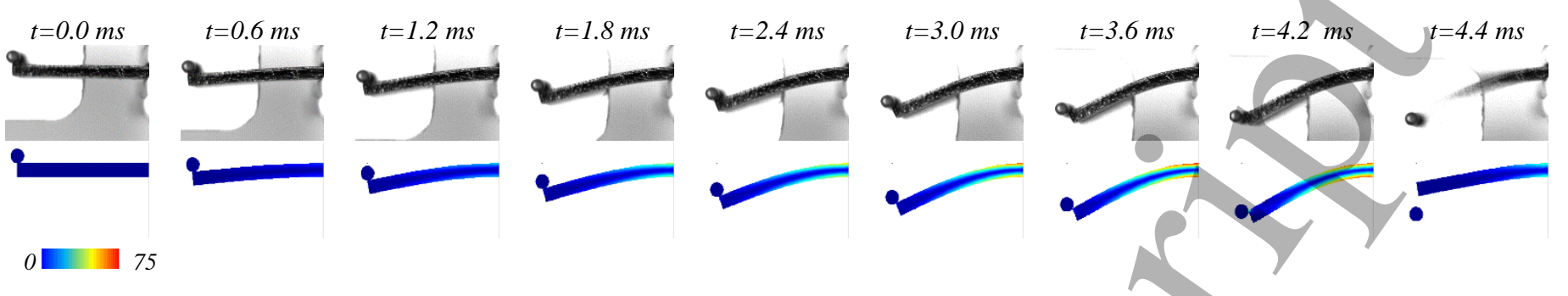

Fig. 20. Configuration of the beam during the forced vibration range $\left(V_{0}=2 \mathrm{~m} / \mathrm{s}, X i=0\right)$. (color bar shows axial stress) 
(a)

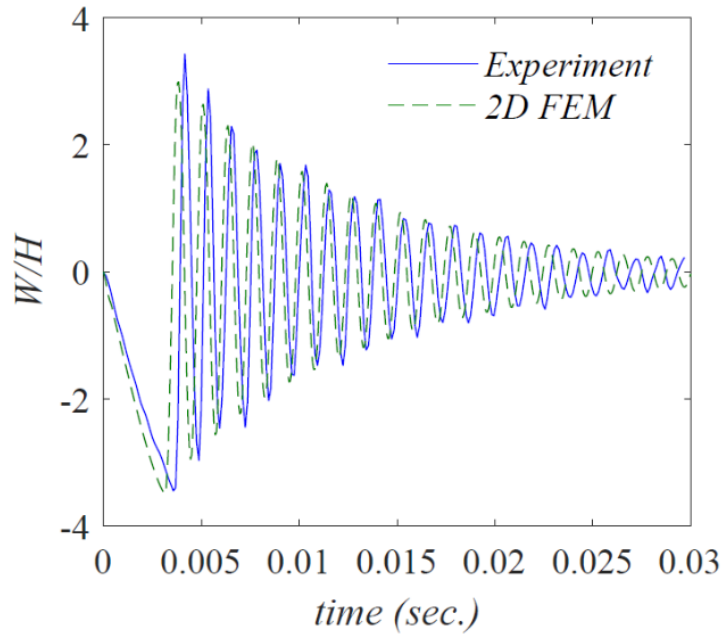

(b)

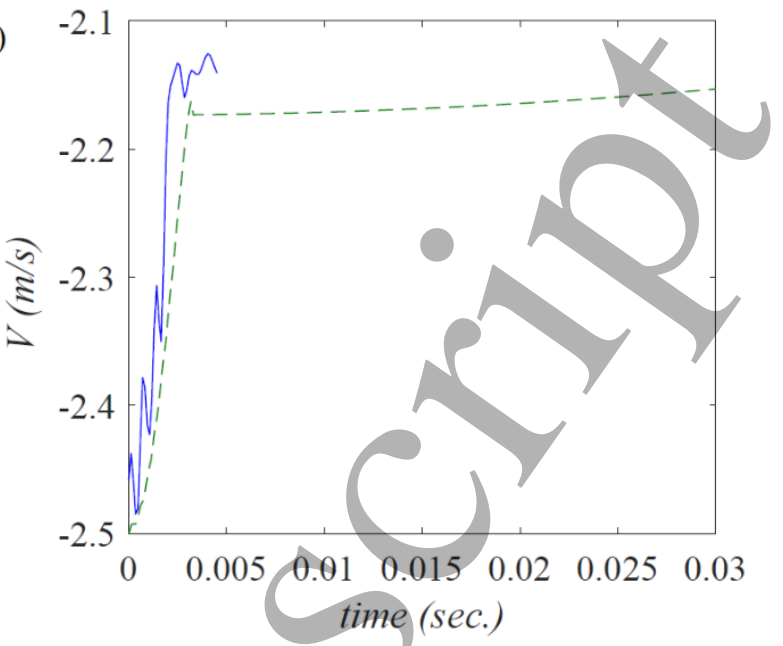

Fig. 21. Time history of displacement (a), impactor velocity (b) of the SMP beam impacted by $V_{0}=2.5 \mathrm{~m} / \mathrm{s}$ at $X i=0$. 
1

2

3

4

5

6

7

8

9

10

11

12

13

14

15

16

17

18

19

20

21

22

23

24

25

26

27

28

29

30

31

32

33

34

35

36

37

38

39

40

41

42

43

44

45

46

47

48

49

50

51

52

53

54

55

56

57

58

59

60 (a)

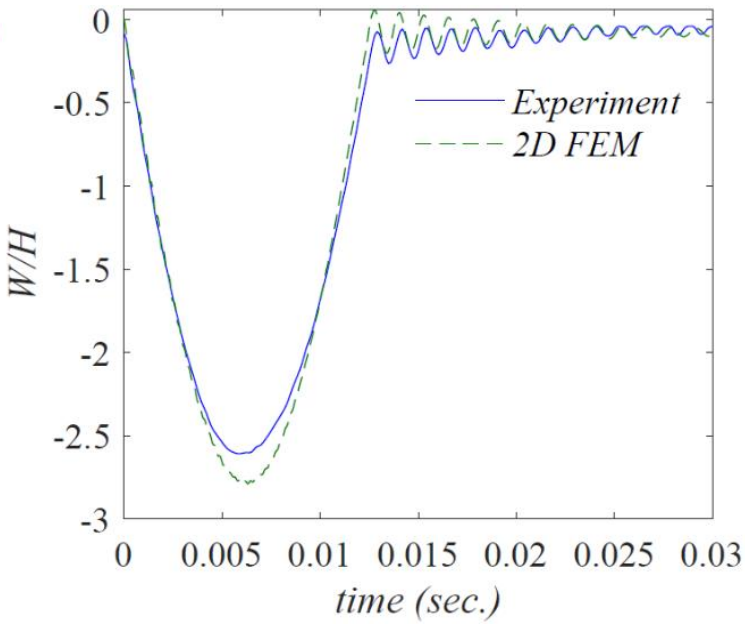

(b)

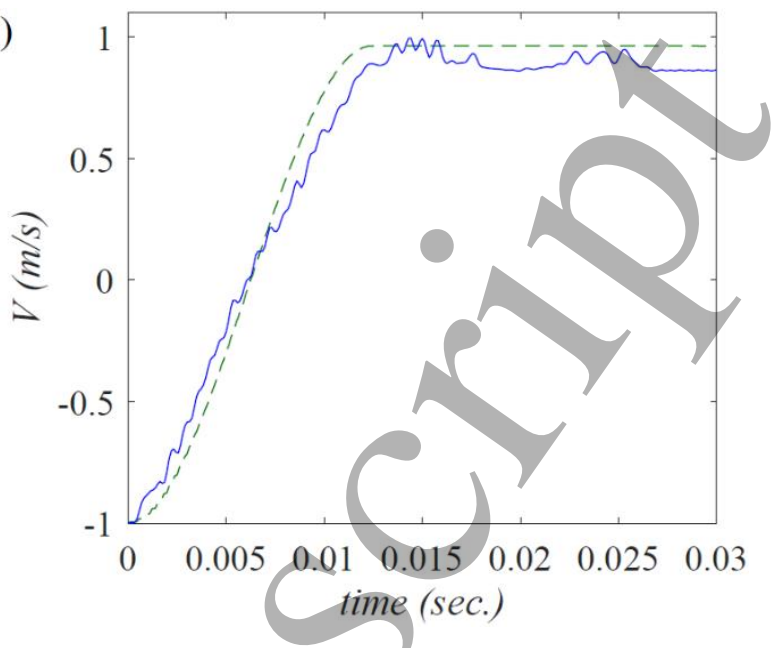

Fig. 22. Time history of displacement (a), impactor velocity (b) of the SMP beam impacted by $V_{0}=1 \mathrm{~m} / \mathrm{s}$ at $X i=\frac{1}{5} L$. 
(a)

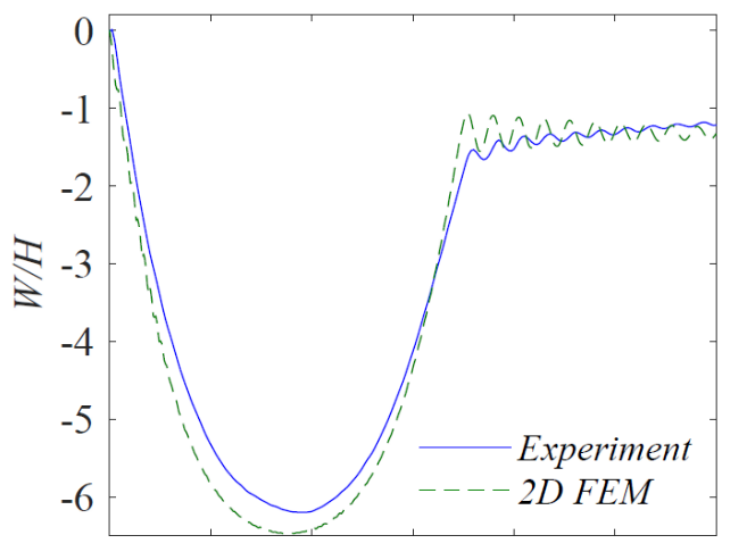

(c)

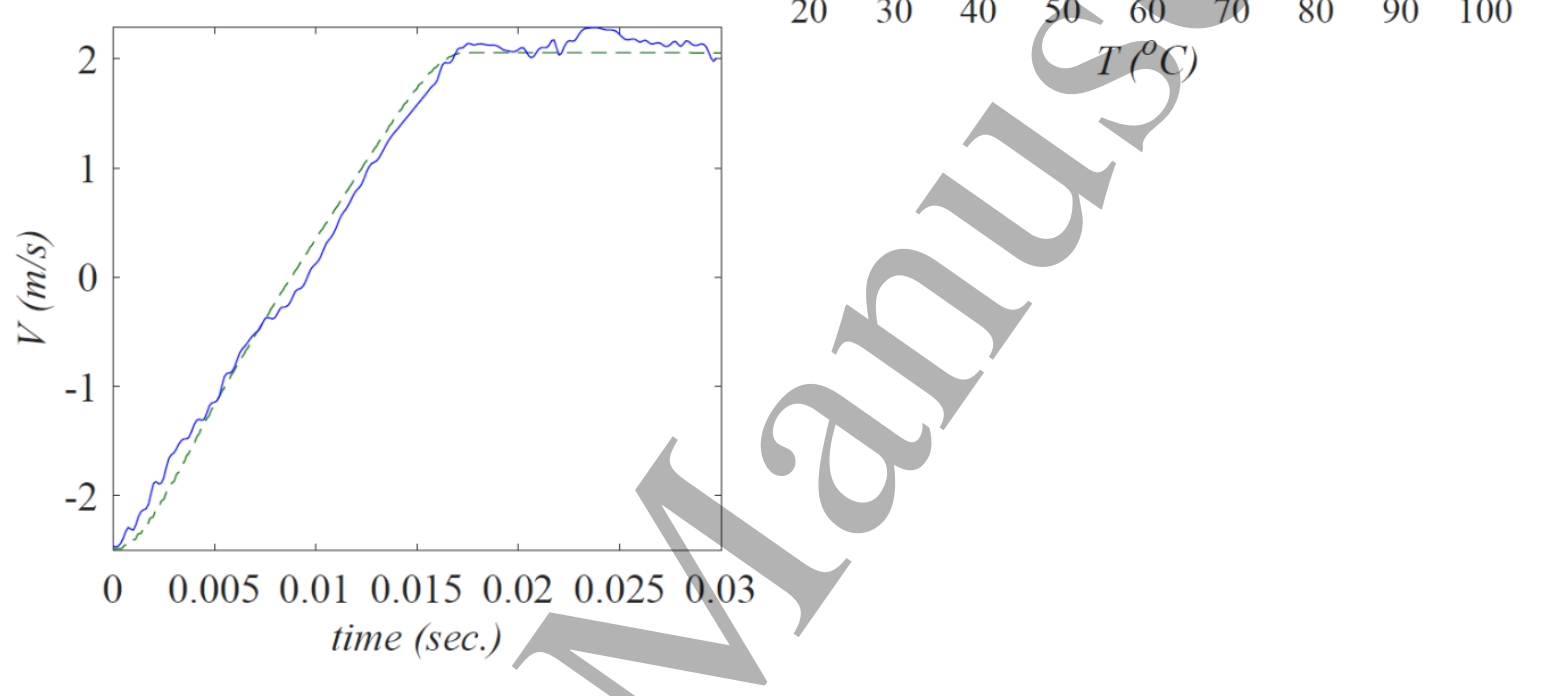

(b)

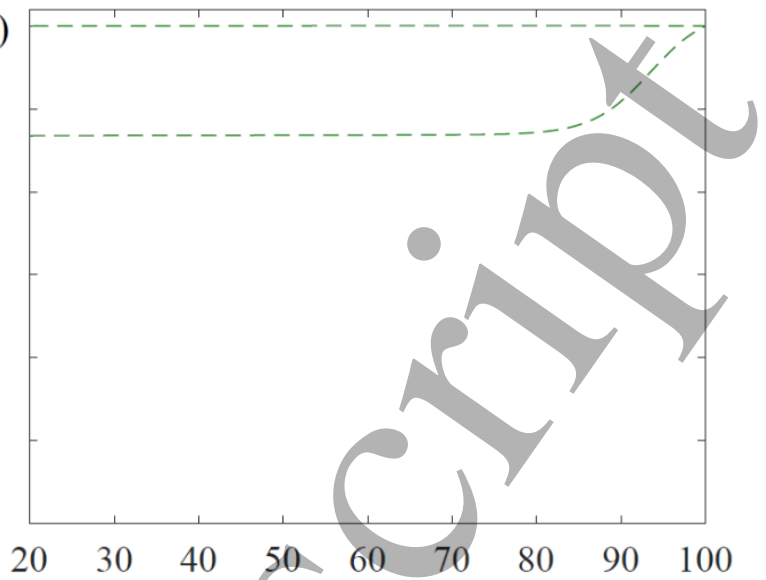

Fig. 23. Time history of displacement (a), impactor velocity (c) of the SMP beam impacted by $V_{0}=2.5 \mathrm{~m} / \mathrm{s}$ at $X i=\frac{1}{5} \mathrm{~L}$. Shape recovery under temperature control (c). 

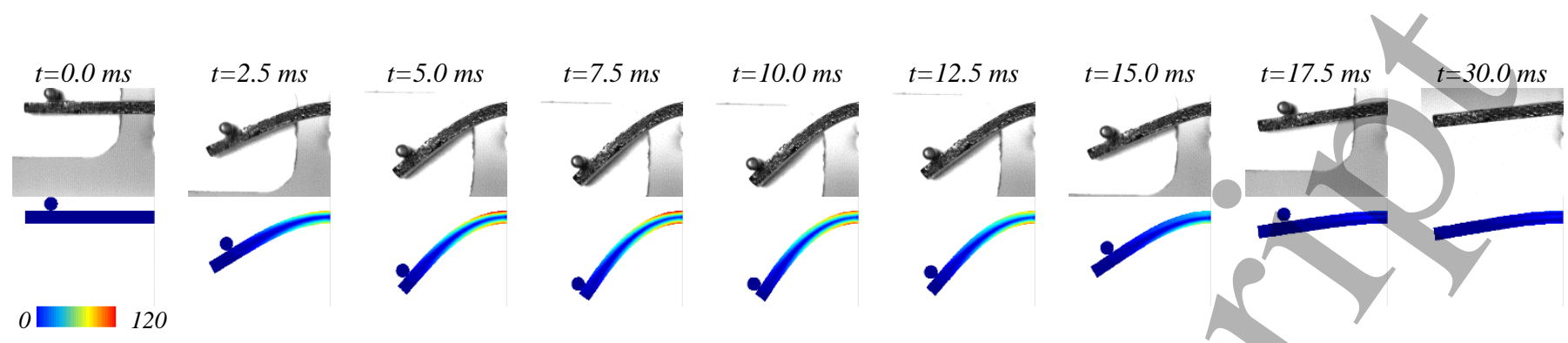

Fig. 24. Configuration of the beam during the forced vibration range $\left(V_{0}=2.5 \mathrm{~m} / \mathrm{s}, X i=\frac{1}{5} L\right)$. (color bar shows axial stress)

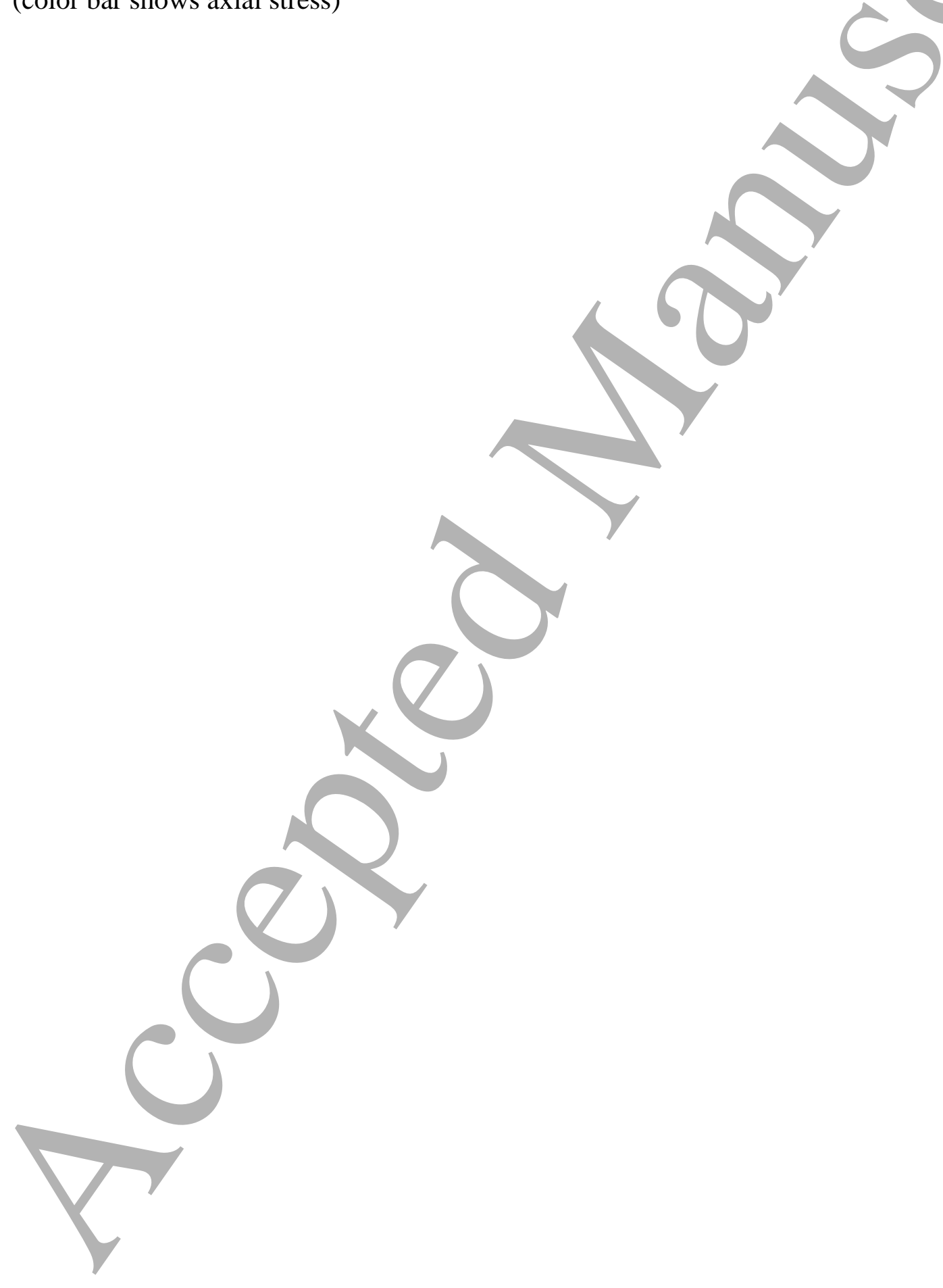


(a)

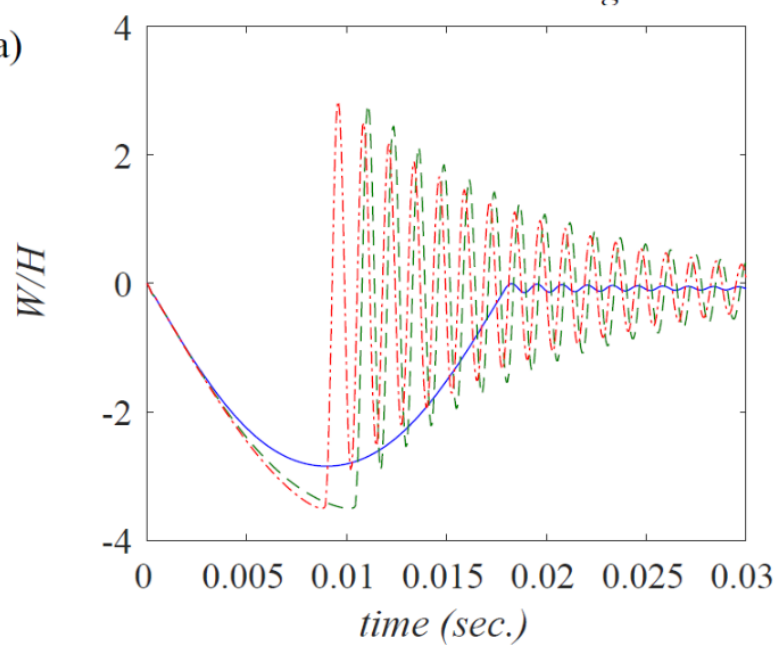

(b)

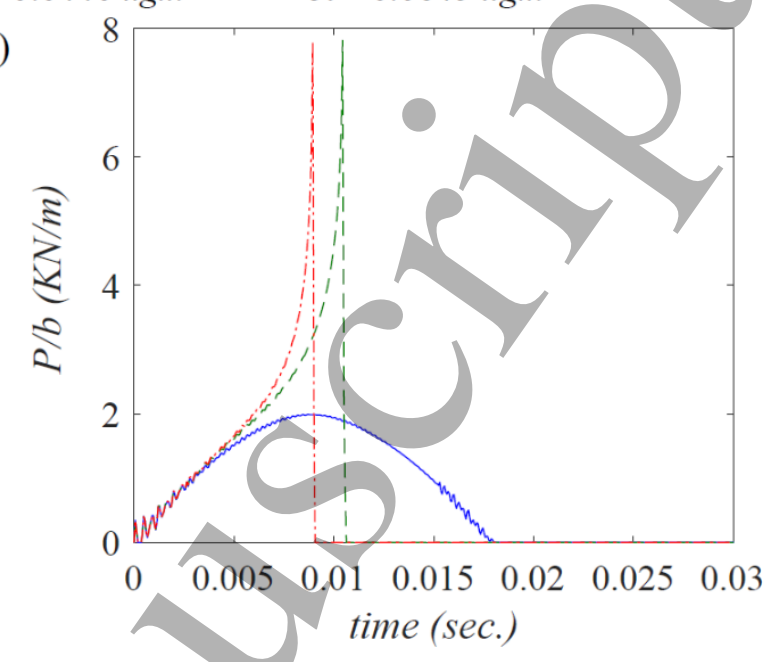

Fig. 25. Time history of displacement (a) and impact force (b) of the SMP beam impacted by $V_{0}=1 \mathrm{~m} / \mathrm{s}$ at $X i=0$ with different moment of inertia. 
(a)

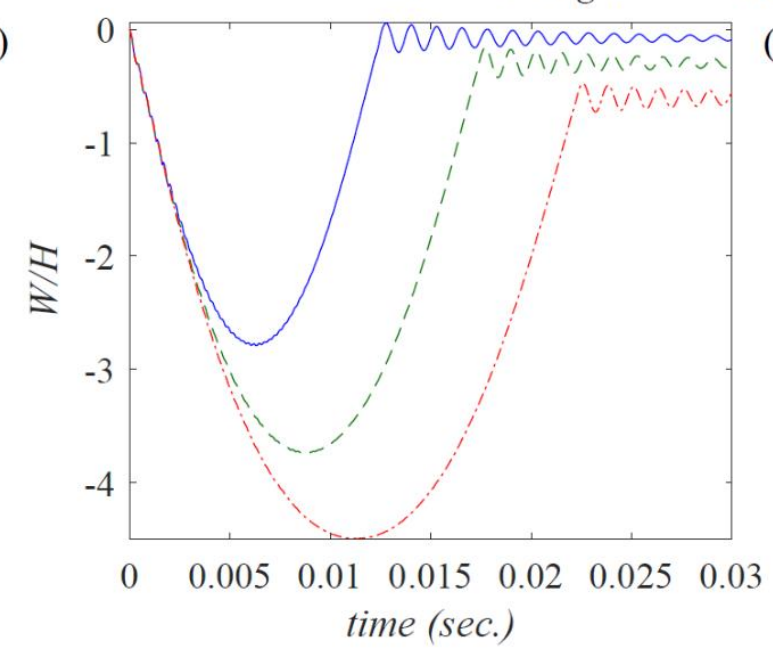

$J i=0.0440 \mathrm{kgm}^{2} \cdots \mathrm{Ji}=0.0645 \mathrm{kgm}^{2}$

(b)

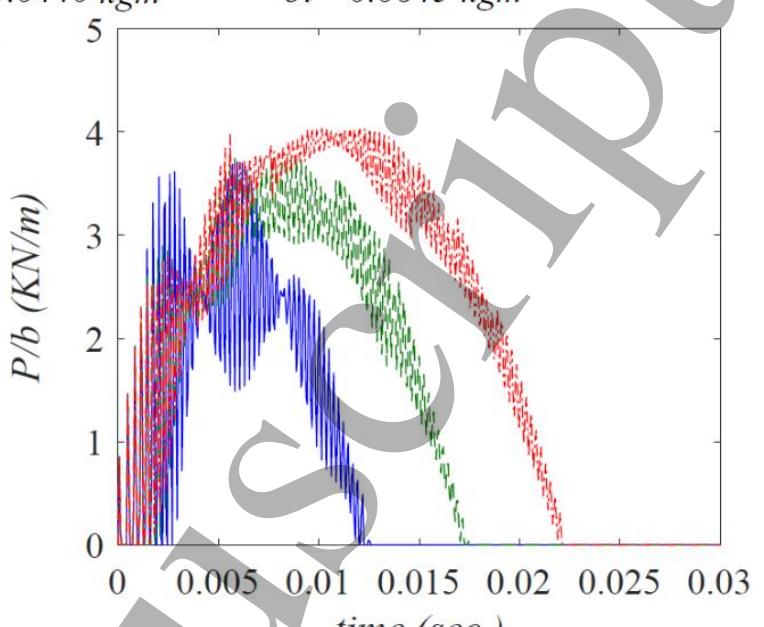

Fig. 26. Time history of displacement (a) and impact force (b) of the SMP beam impacted by $V_{0}=1 \mathrm{~m} / \mathrm{s}$ at $X i=\frac{1}{4} L$ with different moment of inertia. 
(a)

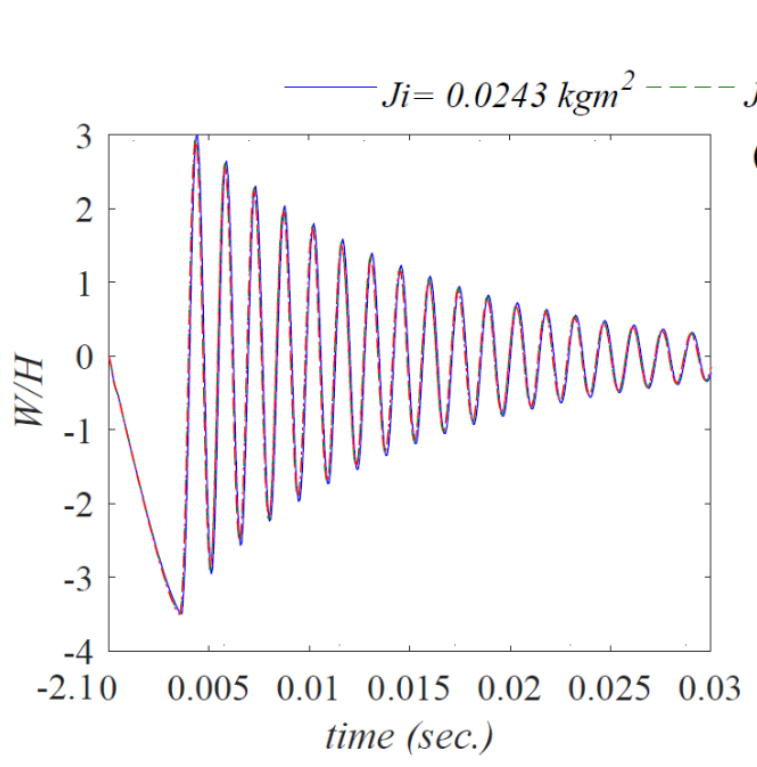

$J i=0.0440 \mathrm{kgm}^{2}-\mathrm{Ji}=0.0645 \mathrm{kgm}^{2}$

(b)

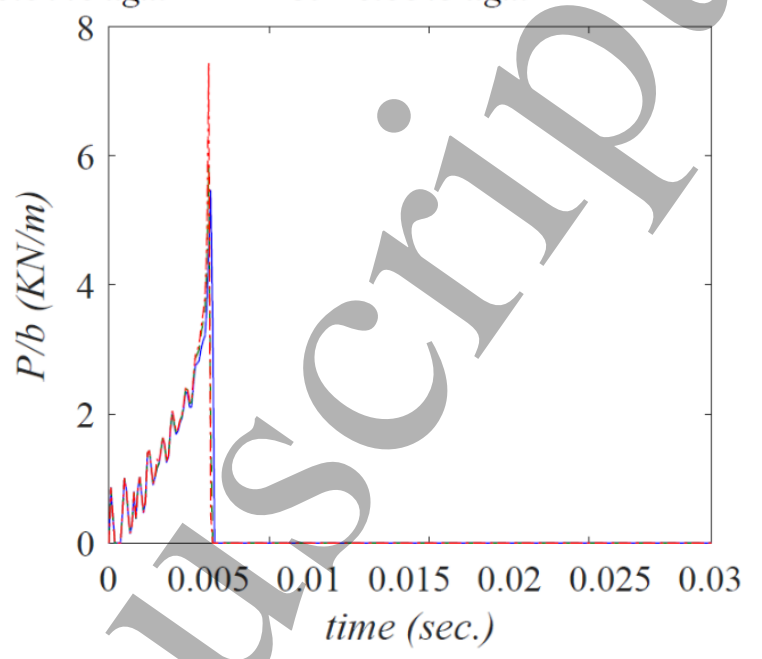

Fig. 27. Time history of displacement (a) and impact force (b) of the SMP beam impacted by $V_{0}=2.5 \mathrm{~m} / \mathrm{s}$ at $X i=0$ with different moment of inertia. 
(a)

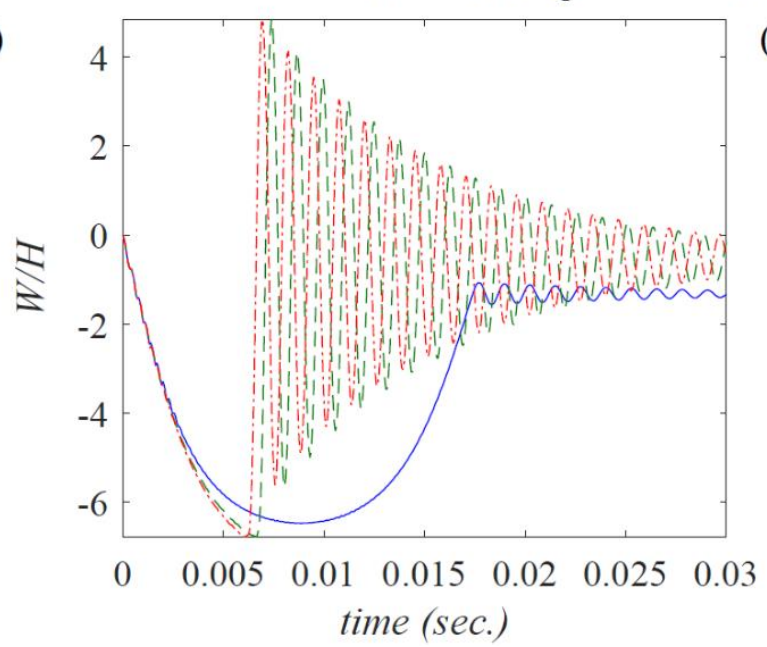

(b)
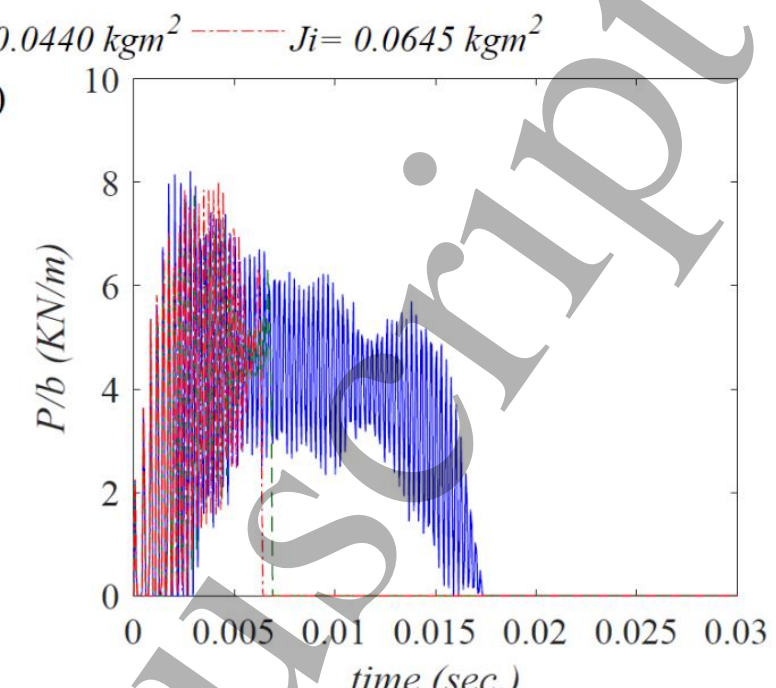

Fig. 28. Time history of displacement (a) and impact force (b) of the SMP beam impacted by $V_{0}=2.5 \mathrm{~m} / \mathrm{s}$ at $X i=\frac{1}{4} L$ with different moment of inertia. 


\section{List of Table}

Table 1. Material parameters from experiments.

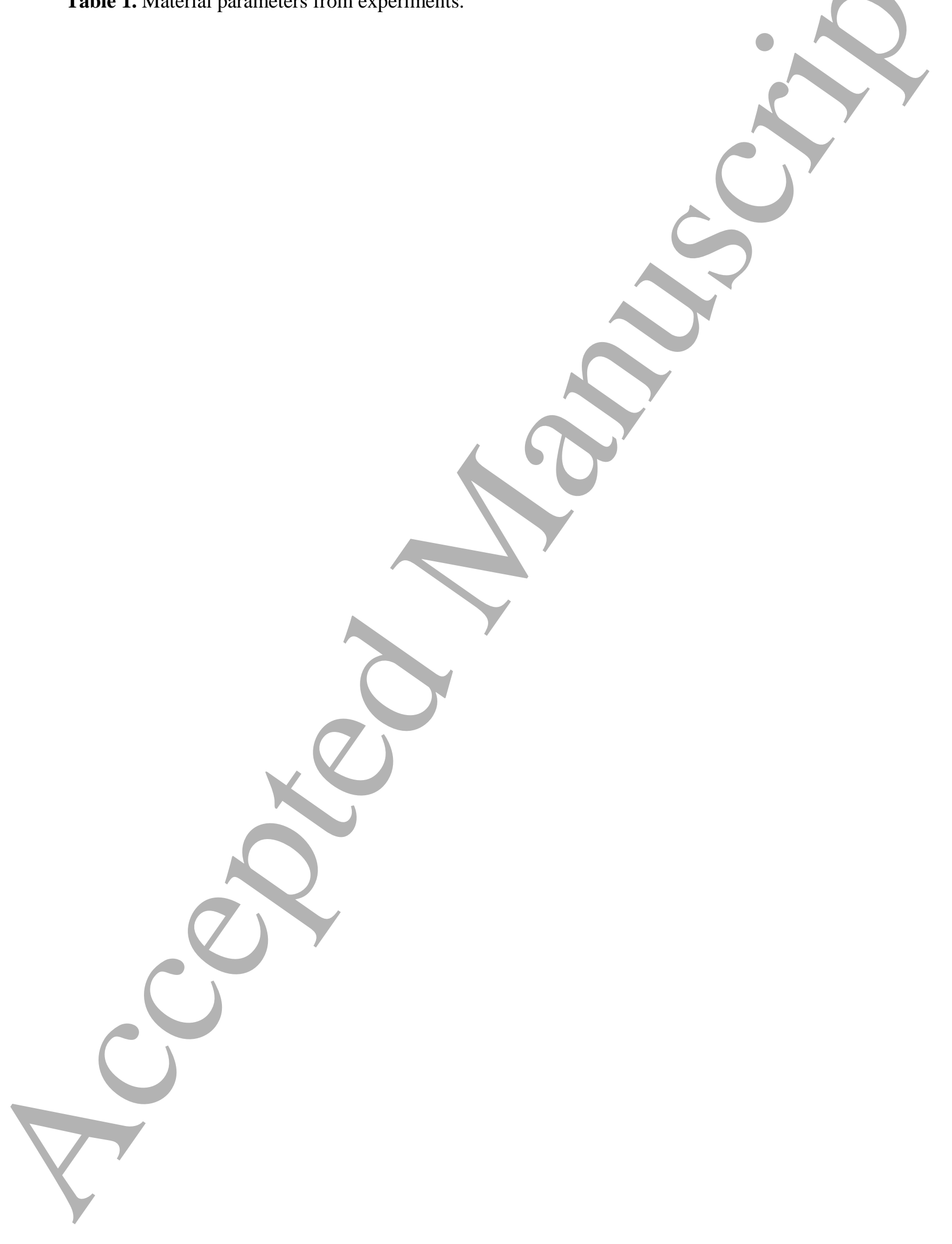


Table 1. Material parameters from experiments.

\begin{tabular}{|cccccc|}
\hline$c_{1 g}(G P a)$ & $c_{2 g}(G P a)$ & $\alpha_{g}\left(10^{-4} K^{-1}\right)$ & $\kappa_{g}(M P a)$ & $c_{g f}$ & $\mu_{g}, \mu_{r}, \mu_{g p}(G P a)$ \\
\hline 0.332 & 0.307 & 1,1 & 1844 & 1 & $0.01,0.01,10$ \\
\hline$h_{g p}(M P a)$ & $Y_{g p}(M P a)$ & $c_{1 r}, c_{2 r}(M P a)$ & $\gamma_{1}, \gamma_{2}$, & $T_{l}, T_{h}, T_{g}\left({ }^{\circ} \mathrm{C}\right)$ & $\rho_{0}\left(\mathrm{~kg} / \mathrm{m}^{3}\right)$ \\
\hline 1 & 23.6 & $0.55,-0.033$ & $0.15,0.145$ & $23,85,60$ & 1500 \\
\hline
\end{tabular}

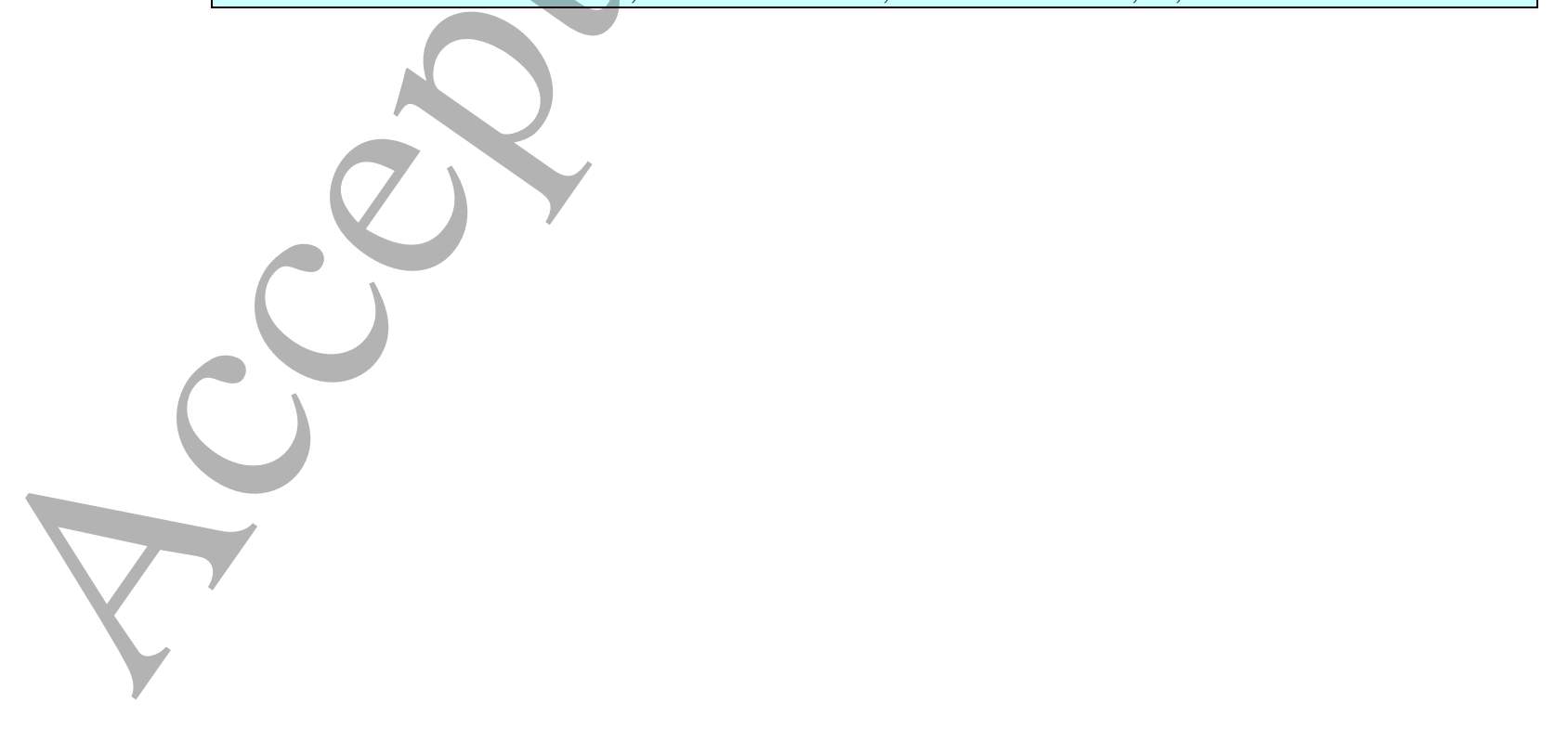

